NASA Contractor Report 189595

\title{
Buffet Test in The National Transonic Facility
}

Clarence P. Young, Jr.

North Carolina State University

Raleigh, North Carolina

Dennis W. Hergert, Thomas W. Butler, and Fred M. Herring

The Boeing Company

Seattle, Washington

Cooperative Agreement NCC1-141

July 1992

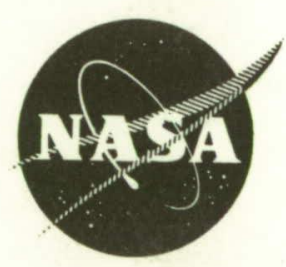

National Aeronautics and

Space Administration

Langley Research Center

Hampton, Virginia 23665-5225

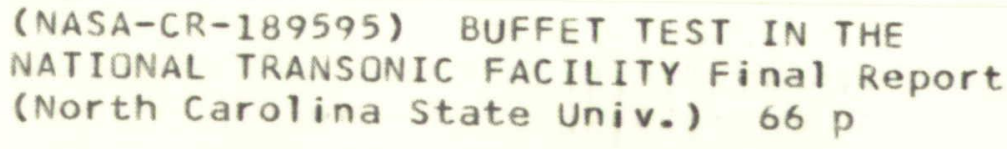


This report documents a buffet test in the National Transonic Facility carried out jointly by the Boeing Airplane Company and the NASA Langley Research Center. The report contains information developed by both Boeing and NASA personnel. The authors wish to acknowledge the contributions of all those personnel at Boeing and Langley who were involved in preparations for the test and actual conduct of the test. The excellent teamwork and dedication resulted in a highly successful test completed in a timely manner. 


\section{TABLE OF CONTENTS}

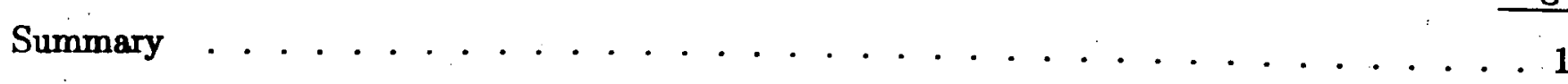

Introduction ...... . . . . . . . . . . . . . . . . . . . . . . . . . 1

Symbols List . . . . . . . . . . . . . . . . . . . . . . . . . . . . . . . . . . . . 1

Model Description . . . . . . . . . . . . . . . . . . . . . . . . . . 2

Test Approach and Run Schedule . . . . . . . . . . . . . . . . . . . . . . . . . . 2

On-Board Instrumentation . . . . . . . . . . . . . . . . . . . . . . . . . . . . . 3

Vibration Monitoring and Model Protection Systems . . . . . . . . . . . . . . . . . 3

Boeing Systems . . . . . . . . . . . . . . . . . . . . . . . . . . 4

NASA Langley Systems . . . . . . . . . . . . . . . . . . . . . . . . . . . . . 4

Transonic Dynamics Tunnel System . . . . . . . . . . . . . . . . . . . . . 4

NTF FFT Analyzer . . . . . . . . . . . . . . . . . . . . . . . . . . . . . . . 5

Balance Dynamic Display Unit (BDDU) and Critical Point Analyzer (CPA) . . . . . . 5

Data Storage . . . . . . . . . . . . . . . . . . . . . . . . 5

Video Monitors . . . . . . . . . . . . . . . . . . . . . . . . . . 5

Monitoring System Location in Control Room . . . . . . . . . . . . . . . . . . . . 6

Wind Tunnel Interlock System . . . . . . . . . . . . . . . . . . . . . . . . . . . 6

Pre-Test Dynamic Monitoring and Safety Systems Shutdown Checkout ～. . . . . . . . . 7

Wind-Off Vibration Tests . . . . . . . . . . . . . . . . . . . . . . . . . . . . . . 7

Safety Analysis and Risk Assessment . . . . . . . . . . . . . . . . . . . . . . . . 8

Structural Integrity . . . . . . . . . . . . . . . . . . . . . . . 8

Fan Protection . . . . . . . . . . . . . . . . . . . . . . . . . . . . 9

Catcher Screen . . . . . . . . . . . . . . . . . . . . . . . . . . 9

Debris Fence . . . . . . . . . . . . . . . . . . . . . . . . . . . . . . . 9

Undesired Events and Risk Assessment . . . . . . . . . . . . . . . . . . . . . . . 9

Results and Discussion . . . . . . . . . . . . . . . . . . . . . . . . . . . . . . 10

Reynolds Number Sweep With Vortex Generators Installed . . . . . . . . . . . . . 10

Reynolds Number Sweep With Vortex Generators Removed . . . . . . . . . . . . . 10

Aeroelastic Stability Trends For Roll Buffet With and Without Vortex

Generators

Mach Number Sweep With and Without Vortex Generators . . . . . . . . . . . . . 12

Mach Number Sweep With VG's Installed . . . . . . . . . . . . . . . . . . . . 12

Mach Number Sweep With VG's Removed . . . . . . . . . . . . . . . . . . . . 12

Aeroelastic Stability Trends For Pitch or Stall Buffet . . . . . . . . . . . . . . . . 12 


\section{TABLE OF CONTENTS}

(concluded)

Conclusions and Recommendations . . . . . . . . . . . . . . . . 13

References ...................... 13

Appendix A. Specifications, Calibration Test Sequence and Measured Sensitivities for Accelerometers Used for 767 Test Program . . . . . . . . . . . . . . . . . 15

Appendix B. Description of Pre-Test Checkout Dynamic Monitoring and Safety

Shutdown Systems . . . . . . . . . . . . . . . . . . . 18

Appendix C. Safety and Risk Assessment Model . . . . . . . . . . . . . . . . . 21

Appendix D. Special Procedure For Model Protection Variable Inputs . . . . . . . . . . 27 


\section{LIST OF TABLES AND FIGURES}

TABLE

II

III

IV

V

VI

TITLE

Run Schedule for 767 Test Program

Summary of Measured Model System Primary Structural Modes and Frequencies

Critical Loading/Failure Locations For Various Vibration Modes

Wing Stress Calculations (Normalized)

Summary of Critical Model Components, Factors of Safety, and Failure Acceptability For $20 \mathrm{~Hz}$ Roll Mode - 100 g's AT Wing Tip

Undesired Events and Risk Assessment

FIGURE

TITLE

Boeing 767 Model in the NTF

Vortex Generator Locations on Right Wing

Test Profile for Investigating Reynolds Number Effect on Buffet

Photo of Model in NTF Illustrating Accelerometer and Strain Gage Locations Illustration of PCB Accelerometer Installation at Outboard Location on Left Wing of Model-View Looking Forward - Not To Scale

Example of Colorgraphics Frequency and Time Domain Display For Roll Buffet Condition

Example of Colorgraphics Frequency and Time Domain Display For Pitch Buffet Condition

Typical BDDU Display

Monitoring System Location in NTF Control Room

Monitoring and Shutdown Systems Schematic

Setup For Dynamic Loads Monitoring and Shutdown Systems Checkout

Wind-Off Vibration Test Setup

Vibration Modes of Primary Interest for Buffet Test

Finite Element Model of Pure Roll Mode $(20 \mathrm{~Hz}$.)

Finite Element Model of First Wing Bending Symmetric Mode $(55 \mathrm{~Hz}$.

Balance Yawing Moment Response Showing Evidence of Fouling Between Aft

Fuselage and Swept Strut. (At Mach 0.80, Re No. $67 \mathrm{million} / \mathrm{ft}, \alpha=4.4^{\circ}$ )

Balance Yawing Moment and Side Force Response From Forced Vibration Tests Mlustrating Fouling Between Aft Fuselage and Swept Strut.

Example Frequency Spectrum for Outboard Wing Accelerometer and Balance Rolling Moment

Roll Buffet Stability Index Versus Angle of Attack for Variable Reynolds Number and with VG's Installed

Roll Buffet Stability Index Versus Angle of Attack for Variable Reynolds Number and with VG's Removed Force Balance and Strain Gage Dynamic Response Due to Roll Buffet Buffet Boundaries for Model at cruise Reynolds Number of 67 Million/ft Force Balance and Strain Gage Response Due to Pitch Buffet 


\section{Summary}

A buffet test of a commercial transport model was accomplished in the National Transonic Facility (NTF) at the NASA Langley Research Center. This aeroelastic test was unprecedented for this wind tunnel and posed a high risk to the facility. This paper presents the test results from a structural dynamics and aeroelastic response point of view and describes the activities required for the safety analysis and risk assessment. The test was conducted in the same manner as a flutter test and employed on-board dynamic instrumentation, real time dynamic data monitoring, automatic, and manual tunnel interlock systems for protecting the model. The procedures and test techniques employed for this test are expected to serve as the basis for future aeroelastic testing in the National Transonic Facility. This test program was a cooperative effort between the Boeing Commercial Airplane Company and the NASA Langley Research Center.

\section{Introduction}

In the fall of 1988, a Boeing 767 transport model was tested in the National Transonic Facility (NTF) at high Reynolds number $\left(R_{e}\right)$. When testing at $R_{e}$ number of 67 million/ft at Mach numbers of $0.75,0.80,0.82$ and at angles-of-attack between 3 to 5 degrees, significant model vibrations were encountered. These oscillations appeared to be a strong function of $R_{e}$ number and Mach number in that these oscillations were observed at Mach 0.80 and $R_{e}$ number of 67 million/ft but not at $R_{e}$ number of 37 million/ft for the same Mach number. Subsequent to this test, analyses were performed in an attempt to understand the cause of these oscillations. Although the exact nature of the flow excitation was not determined, the analyses suggested that the oscillations occurred at angles-of-attack where the lift curve slope breaks, indicating onset of flow separation over the wing. The mechanism is postulated to be a limited amplitude aeroelastic instability involving interaction of the rigid body roll inertia and the balance roll spring, driven by alternating flow separation and reattachment induced by variation of the effective angle-of-attack in rolling motion.

In 1991, a second test of the model was undertaken in order to understand the relation between test variables (Mach number, Reynolds number and dynamic pressure) and model dynamics; develop test techniques and procedures to allow safe testing into the buffet regime; and to verify wing vortex generator performance. (The actual airplane is equipped with two sets of vortex generators near the wing mid-span.) Since the NTF does not have any protection for the fan blades and the stability of the model system aeroelastic (buffet) response could not be predicted with confidence, the re-entry test was judged to be a high risk for the facility. NASA Langley agreed to the re-entry contingent upon an acceptable Safety Analysis Review (SAR).

The objectives of this paper are to describe the analyses, tests, instrumentation, on-line monitoring system, tunnel interlock and test procedures that were employed for this special test in the NTF. Preparatory investigations as well as elements of the failure modes analysis and risk assessment are presented, along with the results of the measured model system aeroelastic response during the wind tunnel tests. The Boeing 767 buffet test is expected to serve as the benchmark for all future aeroelastic and/or other high risk testing in the NTF.

\section{SYMBOLS LIST}

$A \quad$ RMS amplitude of frequency response

$C_{L} \quad$ Lift coefficient

$C_{M} \quad$ Moment coefficient

ft Foot 


$\begin{array}{ll}\mathrm{ft} & \text { Foot } \\ g & \text { Acceleration of gravity } \\ \mathrm{Hz} & \text { Hertz } \\ \text { in. } & \text { inches } \\ \mathrm{kip} & \text { One thousand pounds } \\ \mathrm{ksi} & \text { kips per square inch } \\ K_{1 c} & \text { Measure of fracture toughness } \\ \mathrm{mv} & \text { Millivolt } \\ \mathrm{psf} & \text { Pounds per square foot } \\ q & \text { Dynamic pressure } \\ R_{e} & \text { Reynolds number } \\ \alpha & \text { Angle of attack }\end{array}$

\section{Model Description}

The Boeing 767 model installed in the NTF is shown in figure 1. The model is supported by a swept strut sting and uses an NTF 101B force balance. The model and support structure is constructed of 18 nickel Maraging Steel Grade 200. This material is used extensively for NTF models because of its high strength and acceptable fatigue and fracture properties when exposed to a cryogenic environment. Typical properties at $-250^{\circ} \mathrm{F}$ include an ultimate strength of $252 \mathrm{kips} / \mathrm{in}^{2}$. Young's modulus of 30 million $\mathrm{lbs} / \mathrm{in}^{2}$ and a fracture toughness $K_{1 c}$ of $85 \mathrm{ksi}-\mathrm{in}{ }^{1 / 2}$.

The .03 scale model has 48 pressure orifices, a wingspan of 56 inches, is 56 inches long and weighs approximately 238 pounds. The model has been previously tested in the Boeing Transonic Wind Tunnel and the NASA Ames Research Center 11-foot Transonic Tunnel.

A planform view of the right wing is given in figure 2. In the figure, the locations of the vortex generators (VG's) on the wing are shown. The scaled VG's were bonded to the wings and are extremely small, i.e. .09 in. long, .024 in. high and .004 in. thick.

\section{Test Approach and Run Schedule}

Prior test results showed the roll buffet (buzz) oscillations to be sensitive to Reynolds number. The test approach was to obtain data at various Reynolds numbers and dynamic pressures, at the cruise Mach number of 0.80 , for the configuration with and without vortex generators. This approach resulted in the test program profile shown in figure 3 . Initial high $R_{e}$ number runs were to be made with wing vortex generators on, with the expectation that the severity of the roll buffet model dynamics would be reduced or eliminated. The magnitude of the primary test variables $R_{e}$ and $q$, would then be slowly increased as per the test profile of figure 3 , and small angle-of-attack increments taken as indicated in the run schedule of Table I.

Emphasis was placed on testing the model safely. Reliance was placed on special model dynamics instrumentation, experienced Boeing and NASA LaRC test personnel and wind tunnel interlocks for model/facility protection. Once confidence was established in the instrumentation, monitoring systems and other safeguards, the plan was to test into and (if possible) through the roll buffet regime. After this part of the plan was completed, the model was to be tested with and 
without vortex generators to verify their aerodynamic performance and to complete the rest of the test program according to the run schedule of table $I$.

\section{On-Board Instrumentation}

Safety requirements for testing in the NTF, dictated that special instrumentation be installed on the model and swept strut (sting). During the 1988 test, limited dynamic data from the force balance and a video of the oscillations were used for post-test analyses. Quantitative analyses relative to the elastic response of the wings, swept strut, and the vibration modes involved could not be obtained from the limited data. Therefore, for this test 3 accelerometers were installed in the wings. Also, the wing and swept strut were strain gaged to provide additional data. The additional instrumentation provided capability to monitor the rigid body, and flexible modes of primary interest for the model system. The wings were machined by Boeing for installation of miniature accelerometers which had been qualified and calibrated for cryogenic use. Appendix $A$ gives a description of the accelerometers and the qualification test program that was performed to establish that the accelerometers would work in a cryogenic environment.

The locations of the accelerometers are indicated in the photograph of figure 4. The most outboard accelerometer was located in the left wing about 5 inches from the tip. The other accelerometer placed in the left wing was located at the outboard flap track fairing approximately 10 inches from the wing tip. These two accelerometers were positioned to monitor the wing bending modes of principal interest as well as the model rigid body roll modes. The third accelerometer was located in the right wing at the intersection of the engine nacelle strut with the wing (18.5 inches inboard from the tip). This accelerometer was used to monitor the rigid body roll modes but was not used for wing elastic modes response due to its location at or near node points of the two wing modes of interest (see section on wind-off vibration tests). The two accelerometers in the left wing provided redundancy for monitoring the wing bending modes. Instrumentation redundancy is particularly important for testing in the NTF due to the likelihood that instrumentation will be lost in the harsh environment. Also, model access to troubleshoot or repair instrumentation requires tunnel warm-up which is very costly in time and resources.

The accelerometers were installed in insulated foam cups (see figure 5 for typical installation), to prevent grounding problems. The accelerometer wires were routed in existing or special channels (grooves) machined into the wing. Planned installation of a pressure transducer near the trailing edge on the left wing could not be made due to lack of sufficient space in the channels for both accelerometers and the transducer wires. Problems were encountered with cracking in the channel filler material during the initial cryogenic cycling of the model in the NTF cryogenic test chamber. Subsequently, a filler material consisting of Hysol EA 9309 epoxy, mixed with 100 micron carbon spheres on a 1:1 ratio by volume, was used without problems (see ref. 1 for information on filler materials). During cryo cycling of the model system in the NTF model conditioning chamber, the accelerometers were functionally checked by vibrating the model at various times during the cooldown, cold soak, and warmup cycles.

The NTF 101B balance provided force and moment dynamic data corresponding to the six measured components. The dynamic data from the force balance are processed and monitored by the Balance Dynamic Display Unit (BDDU) and Critical Point Analyzer (CPA) (see ref. 2 for a description of the BDDU and CPA).

\section{Vibration Monitoring and Model Protection Systems}

The primary function of the vibration monitoring and model protection system was to protect the model against failure by limiting the static plus dynamic loads to pre-established limits. The 
system was comprised of both Boeing and NASA equipment and employed equipment and test techniques that are used at Boeing and at NASA LaRC for buffet and flutter testing.

\section{Boeing System}

The Boeing Company uses a portable monitoring and shutdown system called Dynamic Response Actuated Switch (DRAS) which has been used for flutter testing for approximately fifteen years. DRAS has the capability to monitor 2 channels/unit of dynamic data simultaneously. It has provisions for automatically tripping wind tunnel interlock systems if pre-set limit levels (voltage output from on-board instrumentation) and vibration cycle counts are exceeded. DRAS uses lowpass filters for mode separation between channels. By filtering, DRAS can monitor 4 or more different vibration modes (depending on the frequency spread) simultaneously. DRAS monitors time domain signals and employs a trip algorithm which automatically opens or closes a relay switch(es). It has provisions for setting dynamic amplitude levels (voltage output from an accelerometer for example), and cycle count. The cycle count provision limits trips associated with signal spikes and is usually set for 3 to 9 cycle counts. Lights on the DRAS units indicate cycle count and trip status.

In addition to DRAS, the Boeing Co. has developed a Wind Tunnel Monitoring System (WTMS) for real time dynamic monitoring. This system provides real time colorgraphic displays in both the time and frequency domains. This WTMS provides real time diagnostic information which is essential to understanding the character of the aeroelastic response in terms of vibration modes participation, and buildup in amplitude levels as tunnel operating parameters are varied. Also, energy levels and frequency changes are monitored along with other types of information which can be used for initiating manual interlock trips or making risk decisions during a real time test situation. An example of the colorgraphics display used for this test is given in figure 6 . Examples of the WTMS colorgraphics displays used for this test are given in figures 6 and 7 . In the figures, the time domain signal is shown at the top, with the peak values shown in the bar graph in the lower right hand corner as a function of elapsed time. The two displays on the left side of the figures are the frequency versus amplitude (indicated by volts) and the waterfall display which shows the amplitude in color versus time (vertical scale) and frequency. The snapshot display in figure 6 illustrates a roll buffet (buzz) condition where the high vibration levels are associated primarily with the rigid body roll mode at $20 \mathrm{~Hz}$. Wing bending mode responses at $55 \mathrm{~Hz}$ and $77 \mathrm{~Hz}$ respectively, are also present as can be seen in the spectra. Figure 7 illustrates a pitch buffet condition where the high vibration levels are associated primarily with the first sting bending mode at $10 \mathrm{~Hz}$. Also, as can be observed from the figure, the rigid body rolling mode $(20 \mathrm{~Hz})$ vibration is present but at a much lower energy level. The roll and pitch plane vibrations in figures 6 and 7 are representative of run conditions that resulted in the wind tunnel interlocks being tripped to rapidly unload the model. The Boeing DRAS and WTMS were used primarily to monitor the accelerometers placed in the wings which gives a normalized measure of the dynamic loads/stresses at critical (highly stressed) points in the model structure. These systems could have been used to monitor any of the dynamic signals.

\section{NASA Langley Systems}

Systems employed by NASA Langley Research Center (LaRC) for monitoring and protection of the model system are described in the following sections.

Transonic Dynamics Tunnel System. Personnel from the LaRC Transonic Dynamics Tunnel participated in the test and used equipment and techniques that are typically employed for flutter testing in the Langley Transonic Dynamics Tunnel (TDT). This system consists of a Fast Fourier 
Transform (FFT) Analyzer and an 8 channel strip chart or O-Graph. The FFT Analyzer is used to monitor the frequency response of the model system and to track the RMS amplitude of the response for each of the vibration modes of interest as a function of tunnel test parameters. For this test, the inverse of the peak amplitude response (1/A) was plotted at various test points to measure the rate of change of the aeroelastic response and trends toward aeroelastic instability (see ref. 3 for a discussion of the technique).

A strip chart recorder was used to monitor the time domain signal for indications of amplitude buildup and/or trends toward instability. The use of this system by an experienced test engineer provides the capability for interlock trip decisions (manual mode). The combined use of the FFT and the time domain data from the strip chart recorder were used as a basis for aeroelastic response analysis and risk decisions related to test continuation, and/or changes in test variables. The TDT system was used to monitor the three wing accelerometers, two swept strut strain gages, and selected force balance channels, e.g. rolling moment, and yawing moment.

NTF FFT Analyzer. This analyzer was used to monitor selected channels for evidence of fuselage/swept strut fouling, as a backup for the TDT FFT, and for post-test analysis of data from selected runs. In addition, the system provided the capability for immediate playback of any of the data channels being recorded on magnetic tape; in both the time and frequency domain.

Balance Dynamic Display Unit (BDDU) and Critical Point Analyzer (CPA). The BDDU has been used in the NTF for several years to monitor the force balance dynamic loads. The system is described in reference 2. The BDDU monitors the six strain gage balance output channels and provides a visual alarm if the $100 \%$ full scale limit load is exceeded and both visual and audible warning if $140 \%$ of the full scale limit load is exceeded. The full scale limit load is the design load for the individual force or moment component. The alarm algorithm assumes that all other components are simultaneously loaded to $100 \%$ of full scale value. The BDDU is equipped with a bar type display (see figure 8) for each of the six channels and a computer graphics display screen which allows the operator to get snapshot hard copies of the time domain signals for all six or selected channels.

The Critical Point Analyzer scales and sums each normalized signal from the BDDU to obtain the combined static and dynamic signals representative of the loads at pre-determined high stress points. The CPA display is similar to the BDDU display shown in figure 8. A two-level alarm similar to the BDDU is used for this system as well. The CPA was used to monitor and limit the loads on the NTF 101B balance. The unit was coupled to the wind tunnel interlocks which provided an automatic shutdown capability. In addition to preventing overload at critically stressed points on the balance, the CPA was used to limit model side forces and yaw moments which could overstress the swept strut. Both the force balance and swept strut were protected from structural overload by the CPA through the automatic tunnel interlock.

\section{Data Storage}

All dynamic data obtained from the test were recorded on a 14 track magnetic tape recorder. Written records of tape count as well as recorded audio were used to identify and access test points for post-test analyses. These taped data were very useful for post-processing analyses and in particular for analyzing data channels that were not always monitored real time. This magnetic tape is being retained in the NTF archives for future reference. 


\section{Video Monitors}

Two video monitors were located at the Dynamics Monitoring workstation and were used for observing the model dynamics from two different viewing angles. In a normal run situation the video monitors and BDDU serve as the only monitoring devices for model protection.

One camera provided a view of a portion of the fuselage and the left wing. The other view was focused on the left wing tip with the model fuselage discernible in the background. In some cases, particularly for the pitch or stall buffet oscillations, the tunnel interlock(s) was triggered manually based on judgment of the dynamics test engineer observation of the dynamic motion. The combination of viewing the Boeing WTMS, the NASA O-graph and FFT along with the video monitor provided a good real time monitoring system for model dynamics. Also, video cameras were used to assure that the vortex generators remained on the wing during testing. Video tapes of the model dynamics were made for virtually all the runs. Selected runs illustrating the roll buffet and pitch buffet modes of oscillation are available on VHS format.

\section{Monitoring System Location in Control Room}

A planview of the monitoring system layout in the NTF control room is given in figure 9. The Monitoring Workstation included the Boeing WTMS and DRAS systems, the NASA TDT FFT Analyzer and strip chart and the NTF FFT Analyzer station. A block diagram of the Monitoring System workstation is given in figure 10 which illustrates the data acquisition, storage, channels being monitored by specific equipment and associated interlock paths. As previously mentioned, 2 additional video monitors (showing 2 different model views) plus a wind tunnel test parameters monitor were provided at the workstation. The NASA CPA and BDDU stations were located in the Test Conductor's area. The tape recorder was positioned adjacent to the monitoring systems
workstation.

Three manual kill buttons (switches) were located at the workstation for use by both Boeing and NASA personnel. Two additional kill switches were located in the vicinity of the Test Conductor's station. One switch was manned by the NTF Facility Manager or Facility Safety Head while the other switch was installed at the Test Conductor's station. A communication link was established between the workstation and Facility Safety Manager or Safety Head. The Dynamics workstation operator(s) were not in the communication loop with the test conductor and wind tunnel operators in order to avoid communication confusion. Communication from the workstation operator(s) to the Facility Manager or Safety Head on model system dynamics, tunnel parameter changes and/or hold conditions were relayed to the Test Conductor by the Facility Manager or Safety Head in charge of the test.

\section{Wind Tunnel Interlock System}

As previously stated, the monitoring and shutdown system schematic is given in figure 10. As shown in figure 10, the DRAS 1, DRAS 2, and CPA units provided automatic interlock capability for protection of the model structure, force balance and swept strut. Manual interlock switches were provided to both Boeing and NASA personnel.

The National Transonic Facility has the capability for rapidly reducing model loads through the wind tunnel interlock or trip system. Prior to the Boeing 767 test the procedure for unloading the model was to fail-safe the Inlet Guide Vanes (IGV) upstream of the fan and to trip the drive system. The IGV fail-safe unloads the IGV at a rate of 18 degrees/sec which has the potential for 
reducing the test section Mach number from 0.80 to .65 in approximately 2 seconds. This provides a very rapid reduction in dynamic pressure when operating at high Mach numbers (i.e. $>0.65$ ).

Based on earlier tests it was clear that buffet onset was highly sensitive to change in angle-ofattack. (Note: This is generally true for all buffet phenomena related to airfoil separated flow.) A special interlock was installed on the pitch system which provides an angle-of-attack reduction rate of approximately 3 degrees per second.

The interlocks used for the 767 test simultaneously failsafed the IGV and brought the model pitch system to the home position ( 0 angle-of-attack). As previously mentioned, interlocks were set up to be tripped automatically by the DRAS and/or CPA and manual trip (kill) switches were provided to the dynamics test engineers. Also, system functional checks on each automatic channel and manual kill switches were performed prior to each run or test series. The interlock system worked extremely well in rapidly unloading the model with no operational problems encountered.

Pre-Test Dynamic Monitoring and Safety Systems Shutdown Checkout

In preparation for the model entry into the NTF, tests were done to verify operation of monitoring equipment and instrumentation and to perform functional checks for the automated emergency shutdown interlocks. This testing included exercising the Boeing DRAS 1 unit, the CPA, the NASA TDT equipment and NASA NTF FFT Analyzer. This checkout provided an opportunity for the NASA test engineers to become familiar with the Boeing system and to simulate (to the degree possible) an actual run condition. The test setup is illustrated in figure 11. The test is described in detail in Appendix B. The approach was to conduct a forced vibration test to simulate model dynamics during a tunnel run, and exercise the monitoring and shutdown system. Only the DRAS 1 unit was available for this test. The WTMS and DRAS 2 were setup just prior to the actual wind tunnel test. A threaded hole was machined, by Boeing, in the outboard flap track fairing on the right wing to provide an attachment point for the shaker. In addition, the shaker was attached to the fuselage with a special threaded plug which fit into an existing threaded hole
in the fuselage.

\section{Wind-Off Vibration Tests}

To accurately determine the vibration modes that were involved in the model system aeroelastic response, and to set limits on vibration levels during wind tunnel testing, it was necessary to do a wind-off vibration test or modal survey. This test experimentally determines the model system natural vibration modes, frequencies and damping. This information was used to validate the dynamic model of the structure, to evaluate, identify and analyze the system structural mode frequencies during testing, and to set limit load levels associated with the vibration modes of major concern. These data were also used to strategically locate instrumentation (e.g. accelerometers) to assure that the various wing vibration modes could be properly monitored.

The modal survey was performed on the model system after installation in the wind tunnel test section. The test setup is shown in figure 12 which shows the actual model, a vibration exciter system, and modal data acquisition system. A shaker (vibration exciter) was attached to the model at an outboard position on the right wing (as shown in figure 12) and also at the fuselage centerline. The natural vibration data were acquired by an HP 5423A Modal Analyzer System which provides a tabulated set of mode shapes, frequencies and damping data, and displays of the animated mode shapes for different views which are selectable on the machine.

The measured mode shapes and frequencies of primary interest are illustrated in figure 13 . Two of the mode shapes obtained from the Boeing finite element dynamic model are illustrated in 
figures 14 and 15. Note the excellent agreement between calculated and measured frequencies for the roll mode and first wing bending mode. The modes of primary concern for the roll buffet response were the $23 \mathrm{~Hz}$ rigid body roll, 1st wing bending ( $55 \mathrm{~Hz}$ symmetrical) and 1st wing bending ( $77 \mathrm{~Hz}$ asymmetrical). Of particular concern was the potential for getting into a flutter type instability at the wing mode frequencies. Based on the earlier tests and analyses it was thought that the so-called rigid-body roll mode (the model attached to a torsional spring which is represented by the NTF 101B balance) was a clean $20 \mathrm{~Hz}$ rolling motion. However, the wind-off vibration survey revealed two modes that were associated with rigid body roll. For the mode at $19.5 \mathrm{~Hz}$ (see table II and figure 13), the motion is predominantly roll but also involves yawing and pitching of the model on the balance spring. The $23 \mathrm{~Hz}$ mode was a clean roll or torsional oscillation. During the wind-off vibration test, at a resonance frequency of $19.5 \mathrm{~Hz}$ it was experimentally determined that fouling or hard contact occurred between the model fuselage and the swept strut (see figure 4). The minimum measured gap between the model fuselage and swept strut was approximately .32 inches. In the 1988 test an aerodynamic seal was installed in this gap; however for the 1991 tests, no seal was installed in order to simplify the testing. The fact that fouling occurred for the $19.5 \mathrm{~Hz}$ resonance forced response test suggested the likelihood that hard fouling should occur during wind on testing. However, the decision was made not to install the seals, to provide maximum clearance.

\section{Safety Analysis and Risk Assessment}

In order to establish that the Boeing-767 test was an acceptable risk for the NTF, a safety analysis and risk assessment was required. This assessment required a large effort on the part of both Boeing and NASA personnel. The risk assessment model developed for use by Boeing and NASA personnel in performing the safety/risk assessment, is provided in Appendix C. The analyses and tests that were performed to support this assessment are discussed in the following sections.

\section{Structural Integrity}

The Boeing 767 model was one of the first transport models designed for testing in the NTF. The model structural integrity and aeroelastic stability characteristics met the criteria for static testing in the NTF (ref. 4). However, the re-entry for high risk buffet testing required a complete review of the model structural integrity. In-depth dynamic loads analyses were performed to identify all critically loaded components, to establish acceptable dynamic load limit levels and to evaluate fatigue and fracture life. Previously, handbook calculations were sufficient to meet the NASA requirements, but in-depth analysis required a detailed finite element analysis of the wing structure. The control point on the structure (the most highly stressed single order failure point) was found to be the engine nacelle strut to wing attachment holes (wing station 9.3). This was true for the worst case static loads and primary vibration modes including all rigid body roll and elastic wing modes (see Table III). Other areas of concern included fasteners working loose and possible overstress of the swept strut. In table IV, the calculated stresses at the nacelle hole (with and without stress concentration affects) and associated wing tip deflections, for a normalized value of $100 \mathrm{~g}$ 's measured at the wing tip are presented. These calculations reflect only the one particular vibration mode participation for each normalized load case. One significant observation from the data of table IV is that large amplitude oscillations ( 5.5 inches peak to peak) for the $20 \mathrm{~Hz}$ roll mode could be tolerated at an acceptable stress level of $100.8 \mathrm{ksi}$. These results suggested that acceptable model oscillation amplitudes for the $20 \mathrm{~Hz}$ roll mode could be 5 times greater than those experienced in 1988 with the peak stresses well within the dynamic loads allowables of reference 4 . As previously mentioned, the 18 Nickel, 200 grade maraging steel material has an ultimate strength capability of $\sim 250 \mathrm{ksi}$ at $-250^{\circ} \mathrm{F}$. A summary of the critical components, along with their classification and monitoring requirements as determined from the Boeing analysis is provided in table $V$. 
The wing vibration modes were of particular interest due to the potential for wing flutter type oscillations at the higher frequencies (e.g. $55 \mathrm{~Hz}$ and $77 \mathrm{~Hz}$ ). A detailed Boeing finite element model of the wing gave good definition of structural modes and resulted in a high level of confidence for establishing safe dynamic limit loads. All of the detailed structural analyses indicated that the model structure was capable of taking greater dynamic loads than the model balance could withstand for the $20 \mathrm{~Hz}$ roll mode. These results suggested that the force balance would be the limiting structural component for the buffet test, if the $20 \mathrm{~Hz}$ vibration was the predominant buffet mode. However, this did not turn out to be the case and is discussed in the section on "Results and Discussion." Rigid-body roll motion amplitudes of 5 inches (peak to peak) were not considered acceptable either, and reduced limits were used during the test.

\section{Fan Protection}

The National Transonic Facility is not equipped with catcher screen(s) to protect the fan from either a model failure or a failure of any internal structural component in the tunnel. Most facilities which perform dynamic and aeroelastic testing (e.g. the LaRC TDT) are equipped with such catcher screens. Also many closed circuit wind-tunnel facilities have a full set of spare fan blades to allow quick resumption of testing if blades are lost; the NTF did not. Subsequent to the mishap at the NTF in January 1989 (see ref. 5), a replacement set of blades was fabricated which in addition to other structural repairs resulted in a down time exceeding 1 year. Plans were also approved to build a spare set of blades but these blades were not to be ready until mid to late 1991.

Catcher Screen. In preparation for the Boeing 767 test all available means for protecting the fan and other internal components from damage due to potential model failure were investigated. As a result of one of the many design reviews held during the design and construction phase of the NTF, it was recommended and approved that a catcher screen assembly be designed and fabricated for the NTF. This screen was to be located at the end of the high speed diffuser ahead of turn 1 . The catcher screen was an aluminum or steel honeycomb design supported in an egg crate type assembly attached to the tunnel shell. However, installing the screen for the 767 test was determined impractical for the following reasons:

a) The structure was partially fabricated and the steel honeycomb material needed for completion had a very long delivery time (beyond 10 weeks). This could not meet the 767 test schedule.

b) The fabricated parts and pieces would have to be inspected and the installation, fit-up, and tunnel checkout would further lengthen the long lead time.

c) The installation of the screen would add significantly to the critical part count(highly stressed single point failure) for the tunnel.

In the final analysis, it was determined that the screen could not be fabricated, inspected, installed and checked out in time to meet the test schedule.

Debris Fence. An existing debris fence was considered for installation to reduce the risk associated with the test. The debris fence was designed to be installed on the downstream side of the turning vanes in turn 1 but had never been installed during tunnel operations. The installed fence would be three feet from the tunnel floor, and had very little energy absorption capability due to the small wire mesh type construction. Further, some of the welds in the attachment hardware were found to be defective. It was thus determined that the fence had little or no capability for stopping steel parts moving at high speed, and the screen itself posed an additional hazard to the facility. Therefore, the debris screen installation was abandoned. 


\section{Undesired Events and Risk Assessment}

The final step in the safety analysis and risk process (see Appendix C) is to formulate a list or table of undesired events/failure modes, and identify the effects or consequences of such events as well as establish safeguards to eliminate or to reduce the risks to acceptable levels. A summary of the primary undesired events and risk assessments is provided in table VI.

As an example of an undesired event, consider the case when incorrect limits could be entered on the monitoring system (e.g. Boeing DRAS). In order to safeguard against this possibility, a special procedure and Facility Safety Head Approval Form was implemented for this test. This procedure required instrumentation and interlock check-out prior to each run and required both Boeing and NASA personnel to agree on load limit levels and to independently check the trip level settings. The special procedure forms used for the test are provided in Appendix D.

\section{Results and Discussion}

The results of the buffet test are presented in this section. Emphasis is placed on discussion of test techniques, experience and conditions under which buffet was encountered during the test.

\section{Reynolds Number Sweep With Vortex Generators Installed}

The first configuration tested had the vortex generators installed on the model. The test plan run profile shown in figure 3 was followed and testing began on January 18, 1991. During the initial phase of the testing, small angle-of-attack increments $\left(\Delta \alpha \simeq 0.1\right.$ to $\left.0.2^{\circ}\right)$ and conservative limit amplitude (load) levels were used while monitoring the onboard accelerometers and strain gages. Several trips were initiated (either automatic or manual) during the early phases of the test until the model dynamics were characterized and acceptable levels of vibration were established, to avoid premature interlock trips. As the Reynolds number sweeps began, it became apparent that angle-of-attack would be limited to about 5 degrees due to pitch or stall buffet dynamics. Initial testing was slow due to the use of small angle-of-attack increments and the dwell time required for FFT analysis which developed amplitude or inverse amplitude $\left(\frac{1}{A}\right)$ plots as angle-of-attack was slowly increased. The test profile of figure 3 was completed without encountering roll buffet, with VG's on the model. An additional run at Mach .86 and 67 million/ft Reynolds number was completed, with VG's installed, without roll buffet onset. This initial test series was interrupted by the failure of the liquid nitrogen pump on $1 / 25 / 91$. The pump was repaired at LaRC, and testing resumed on $2 / 13 / 91$.

\section{Reynolds Number Sweep With Vortex Generators Removed}

The second series of tests with the vortex generators removed began at a Reynolds number of 49 million per foot since the clean wing configuration (without VG's) was tested previously in 1988 at $R_{e}$ number of 7 and 35 million per foot without buffet encounter. Roll buffet was first observed for this series of tests at $R_{e}$ number of 59 million/ft at Mach .80 . Subsequent testing at Mach .80 and 67.5 million/ft $R_{e}$ number and dynamic pressure of $2670 \mathrm{lb} / \mathrm{ft}^{2}$ resulted in a strong roll buffet response at $\alpha \cong 4.5^{\circ}$. This test repeated the roll buffet encountered with the clean wing model at the same test conditions in 1988. The roll buffet (or buzz) amplitude was about 1 inch peak to peak at the wing tips and the frequency response was predominantly $20 \mathrm{~Hz}$. These data clearly indicated that under roll buffet conditions the fuselage and swept strut were coming into contact (see figure 16) due to the yawing motion associated with the $20 \mathrm{~Hz}$ model response. The fouling occurred at about $80 \%$ of yawing moment full scale and is evident by signal clipping of the response peaks as shown in figure 16. The same type of signal clipping was observed during pre-test 
dynamic load simulation (Appendix B) when fouling was visually observed and recorded as shown in figure 17. This fouling indication during testing was verified by inspection during tunnel entry for model change, and was subsequently monitored closely throughout the test program.

\section{Aeroelastic Stability Trends For Roll Buffet With and Without Vortex Generators}

An established technique for conducting flutter and buffet tests is to develop inverse amplitude $\left(\frac{1}{A}\right)$ plots for the pertinent model frequency responses (FFT of time domain response) as tunnel parameters are varied (see ref. 3). In flutter testing, Mach number and dynamic pressure are normally varied to identify flutter boundaries. In the initial tests series in NTF for varying $R_{e}$ and $q$, Mach number was held constant at 0.8 and angle-of-attack was varied for the model with and without VG's. As a result, the primary variables affecting the buffet response onset and amplitude buildup were angle-of-attack and dynamic pressure. To evaluate trends toward buffet instability, RMS amplitudes were measured with the FFT Analyzer for the structural response modes of interest and the inverse of these amplitudes was plotted as a function of angle-of-attack and Reynolds number for constant Mach number. An example of a typical frequency response spectrum for the 767 model is given in figure 18. In the figure, the upper spectrum is for the left wing tip accelerometer in $g$ 's versus frequency. For the data shown, the predominant modes are at $10 \mathrm{~Hz}$ (1st sting bending mode); $20 \mathrm{~Hz}$ (roll/yaw mode) and $55 \mathrm{~Hz}$ (1st wing bending mode). The bottom graph illustrates the response as observed from the rolling moment output of the force balance. In this case, the predominant balance response frequency is $20 \mathrm{~Hz}$ as this channel cannot detect the structural modes response of the model support structure and the wing.

The 1/amplitude plots for the $20 \mathrm{~Hz}$ mode response at Mach .8 and varying Reynolds number are illustrated in figures 19 and 20 . Figure 19 gives the stability trends for the initial series of tests with the VG's installed on the model, while figure 20 gives similar results with VG's off the model. The condition for incipient instability is when $\frac{1}{A} \rightarrow 0$. As the slope of the curve tends to become steeper and $\frac{1}{A}$ shows a sharp trend toward zero, more data points must be taken at smaller angle-of-attack increments.

In figure 19 , the $\frac{1}{A}$ plots tend to break over at about $3.5^{\circ}$ angle-of-attack. (This corresponds to a break in the $C_{M}$ and $C_{L}$ curve for the model). However, the plot shows that for the $20 \mathrm{~Hz}$ response, although increasing ( $\frac{1}{A}$ decreasing) up to 5 degrees, there is no sharp trend toward instability. As previously stated, roll buffet onset (lock-in) did not occur for these series of runs with the VG's installed. Also, as previously mentioned, these tests were limited to an angle-of-attack of 5 degrees due to onset of stall (pitch) buffet dynamics.

Similar plots of the $\frac{1}{A}$ stability index are given in figure 20 for varying $R_{e}$ number versus angleof-attack without VG's installed. The breakover point also occurs around 3 degrees angle-of-attack but with a much more rapid buildup in amplitude with increasing angle-of-attack. However, for a $R_{e}$ number of 49 million/ft and $q$ of $1962 \mathrm{psf}$, the $20 \mathrm{~Hz}$ mode $\frac{1}{A}$ value decreased slightly between $3.5^{\circ}$ and $4.1^{\circ}$ but became stable (tends toward increasing stability) with increasing angle-of-attack. However, at 59 and 67 million Reynolds number, buffet onset or lock-in is encountered and interlock trips initiated at fairly high (small $\frac{1}{A}$ ) amplitudes. As previously mentioned, because of fouling between the aft model fuselage and swept strut support, attempts to further test into and possibly through the buffet region were abandoned due to risk of structural damage to the model and/or swept strut. Thus, it remains to be determined whether or not the model roll buffet is a limit cycle oscillation. 
An example of the roll buffet response, as seen from the time domain strip chart signal, is given in figure 21. The four channels of data shown in the figure include the yawing and rolling moment response as obtained from the balance and the swept strut bending and torsion strain gages response. When buffet onset and lock-in occurs, the frequency on all channels stabilizes in at $20 \mathrm{~Hz}$ with amplitude increasing rapidly. The buildup in amplitude to the interlock trip point for this case occurred in about 2 seconds. However, note the very rapid decay of the response from peak value to near zero in approximately $1 / 4$ second. Since the tunnel Arc Sector pitch system moves about 3 degrees/second, the data suggests that only a very small change in $\alpha$ relieves the roll buffet lock-in oscillations. The interlock trip for the run shown in figure 21 was initiated manually by the engineer observing this strip chart data.

\section{Mach Number Sweep With and Without Vortex Generators}

After the roll buffet investigation was completed, the next phase of testing focused on obtaining aerodynamic data at a Reynolds number of 67 million/ $\mathrm{ft}$ for varying Mach number with and without vortex generators installed. These tests were conducted in a normal testing mode, that is to say, polars were run at pre-established Mach numbers using normal angle-of-attack sweeps. The function of the team monitoring the dynamic and aeroelastic response would be to trip the interlocks if the pre-established buffet amplitudes were exceeded or roll buffet lock-in was encountered. Once the interlocks were tripped during a Mach number run, the tunnel conditions were changed and the next Mach number run was initiated. It was expected that angle-of-attack would be limited by either roll buffet or pitch buffet, for all polars to be run.

Mach Number Sweep With VG's Installed. The initial run was made at Mach .86 and aerodynamic data were obtained up to $6.5^{\circ}$ angle-of-attack. The interlock automatically tripped on the $55 \mathrm{~Hz}$ mode (1st wing bending) which is believed to be due to system stall response rather than wing flutter. Tests at Mach numbers $.86, .84, .82$ and .80 were limited by pitch buffet. These boundaries are plotted in figure 22. Roll buffet was encountered with VG's on at Mach numbers of 0.75 and 0.70 at angles-of-attack of $5.9^{\circ}$ and $6.7^{\circ}$, respectively. These test results indicated that the VG's altered the flow over the wing such that roll buffet was not encountered at test Mach numbers greater than .75 but the pitch buffet boundary is about the same with or without VG's installed as can be seen in figure 22 .

Mach Number Sweep With VG's Removed. The initial run was made at Mach 86 and data were obtained up to $6.25^{\circ}$ angle-of-attack. This run was limited by a violent pitch buffet response which is discussed in the next section. Roll buffet was observed during the Mach 0.84 run but testing continued through this condition (vibration levels were within limits) to the point where the run was terminated due to pitch buffet at an angle of attack of approximately $6.6^{\circ}$. The remaining runs at Mach $0.82,0.75$, and 0.70 were limited by the roll buffet oscillations.

\section{Aeroelastic Stability Trends For Pitch or Stall Buffet}

As previously mentioned, pitch or stall buffet dynamics limited testing the model at high angles of attack. The pitch buffet boundary is illustrated in figure 22 for tests at full scale Reynolds number (67 million/ft). Buffet at high angles-of-attack is a common occurrence in wind tunnel testing. The problem is usually associated with flow separation over the wing at high angles-ofattack resulting in unsteady aerodynamic loads on the model. Typically, the lowest fundamental model system structural mode in the pitch plane is excited (along with higher modes at lower energy) and amplitude is usually dynamic pressure dependent. High angle-of-attack buffet is not unique to the NTF. However, the loads associated with very high dynamic pressures (e.g. 767 tested at $\sim 2800 \mathrm{psf}$ ) can result in violent, high amplitude oscillations. 
During the full scale $R_{e}$ number and Mach number sweeps with and without VG's attempts were made to get to the highest angle-of-attack possible without jeopardizing structural integrity of the model. The quality of the aerodynamic data was adversely affected when the model vibrated at high angles-of-attack. While testing at Mach 0.86 and 0.84 violent pitch oscillations were encountered at angles-of-attack of 6.4 and 6.0 degrees respectively. During the Mach .86 run the model oscillations were measured to be approximately 2.5 inches peak-to-peak at a frequency of $10 \mathrm{~Hz}$, (1st model/sting bending mode in the pitch plane). At the peak amplitude the left wing inboard accelerometer and swept strut strain gage signals were lost. The peak pitch buffet response at Mach .86 as measured from the balance pitch, roll and normal force output and the bending gage on the swept strut is illustrated in figure 23. Note that the normal force response locks in at $10 \mathrm{~Hz}$ while the pitching moment responds at 10 and $17 \mathrm{~Hz}$ (balance pitching mode). The signal from the swept strut bending gage was lost. After disassembly of the model, it was found that the swept strut strain gage wires were severed due to fouling between the bottom of the swept strut and the bottom of the model fuselage. Figure 23 shows that after the interlock is tripped, the motion is completely decayed in approximately one second. Although the pitch or stall buffet oscillations were violent, the model and support system (sting) were not in jeopardy as the stresses (both combined static and dynamic) were within acceptable limits.

\section{Conclusions and Recommendations}

Buffet testing of the Boeing 767 wind tunnel model was successfully completed in the National Transonic Facility at the NASA Langley Research Center. A comprehensive safety analysis and risk assessment program was carried out by Boeing and NASA personnel, as a prerequisite for testing in the NTF facility. On-line dynamic signal monitoring of on-board instrumentation and wind tunnel interlocks were successfully used to protect the model system. Buffet boundaries were established at various Mach numbers and angles-of-attack at full scale Reynolds number. Pitch or stall buffet limited testing to a maximum angle-of-attack of 6.5 degrees. Testing into the roll buffet region was limited due to fouling between the model fuselage and swept strut support sting. Test techniques employed were based on those used by Boeing and NASA LaRC for testing flutter models.

This test is expected to serve as the model for future aeroelastic tests in the National Transonic Facility. Buffet of wind tunnel models is expected to be a continuing problem when testing in the NTF, at full scale Reynolds numbers and associated high dynamic pressures. It is recommended that pre-entry assessment of the potential for buffet should be made for each model to the tested in the NTF. Also, provisions should be made for instrumenting potentially high risk cryogenic test models with accelerometers or strain gages placed at strategic locations to monitor and protect the model structure against dynamic and/or aeroelastic response overloads.

\section{References}

1. Young, Clarence P., Jr.; and Gloss, Blair B.: Second Workshop on Cryogenic Wind-Tunnel Models. NASA Conference Publication 3010. November 1987.

2. Ferris, Alice T.; and White, William C.: Monitoring Dynamic Loads on Wind Tunnel Force Balances, ISA Paper 89-0021, 1989.

3. Rublin, C. L.; Watson, J. J.; Ricketts, R. H. and Doggett, R. V., Jr.: Evaluation of Four Subcritical Response Methods For One-Line Prediction of Flutter Onset in Wind Tunnel Tests. Journal of Aircraft Volume 10, October 1983, pp. 835-840. 

4. Anon: Wind-Tunnel Model Systems Criteria: NASA Langley Research Center, LHB 1710.15.
August 1986.

5. Report of the NASA-LaRC National Transonic Facility Mishap Investigation Board, Vol. I of III Final Report, April 11, 1989. 


\section{Appendix A \\ Specifications, Calibration Test Sequence and Measured Sensitivities For Accelerometers Used For 767 Test Program}

Specifications for the PCB quartz accelerometers used for the Boeing 767 test program are available from the manufacturer. These miniature accelerometers are about the size of a pencil eraser (.23 in. diameter $\times .30$ in. high) and were tested extensively at the NASA Langley Research Center. The Model No. of the accelerometers used is the 309M42: which has a different type of connecting wire than the 309M15. The accelerometer calibration test sequence is provided in Figure A-1. The test sequence is designed to characterize a cryogenic test profile for the NTF. Accelerometer sensitivities at low temperatures for four of the units are provided in figure A-2. The data of figure A-2 were used to select the sensitivity at test temperature for signal calibration. Note that sensitivity curves slopes are fairly flat for the left wing outboard and right wing nacelle accelerometers at $-250^{\circ} \mathrm{F}$ (test temperature). The accelerometers performed very well during the tests. Drop out of accelerometer signals occurred twice during the test and both were attributed to wire breakage due to thermal and/or mechanical loading. Care must be taken to install the wires to prevent fraying across sharp edges and allow enough slack for wire contraction. 


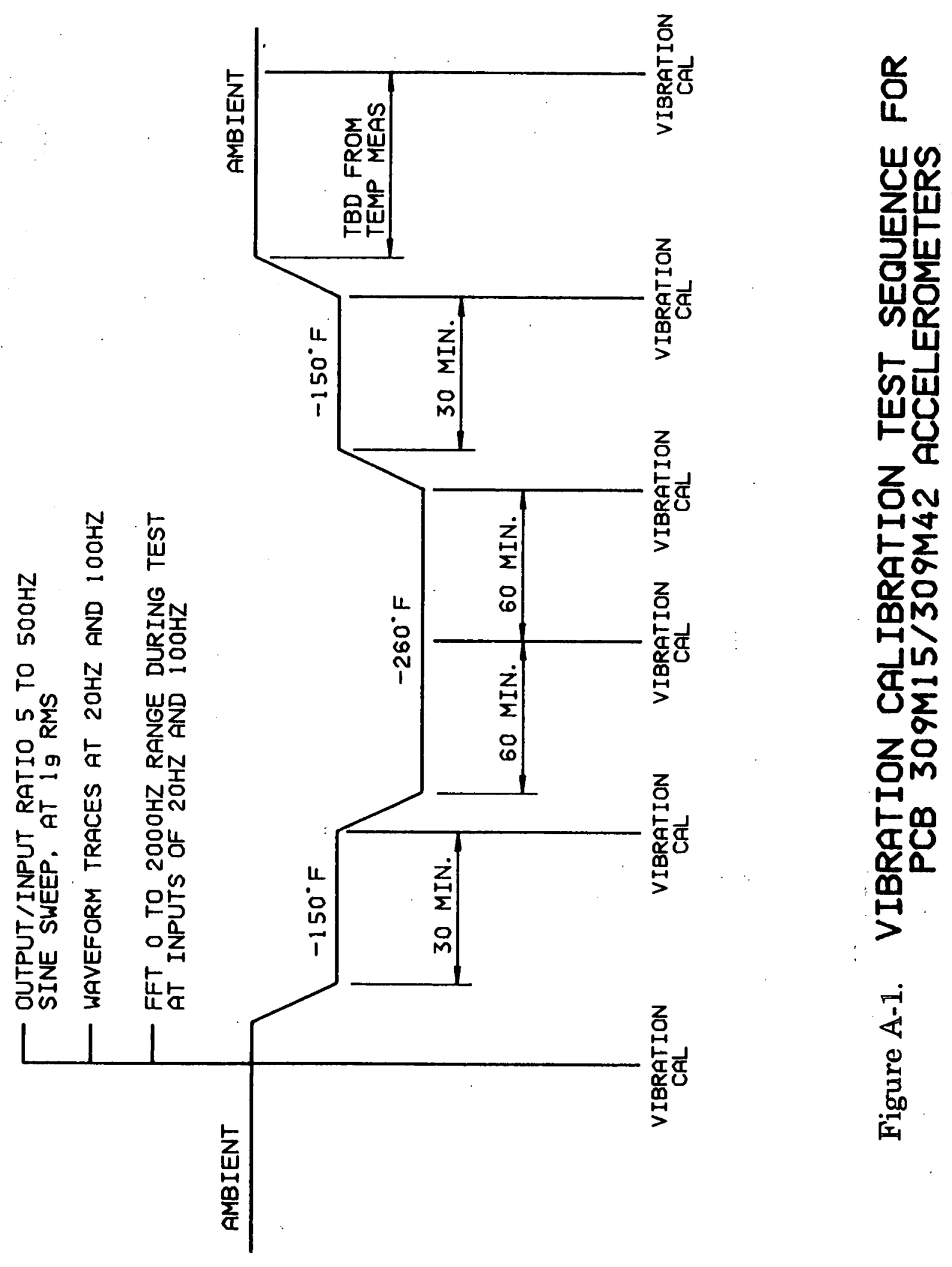



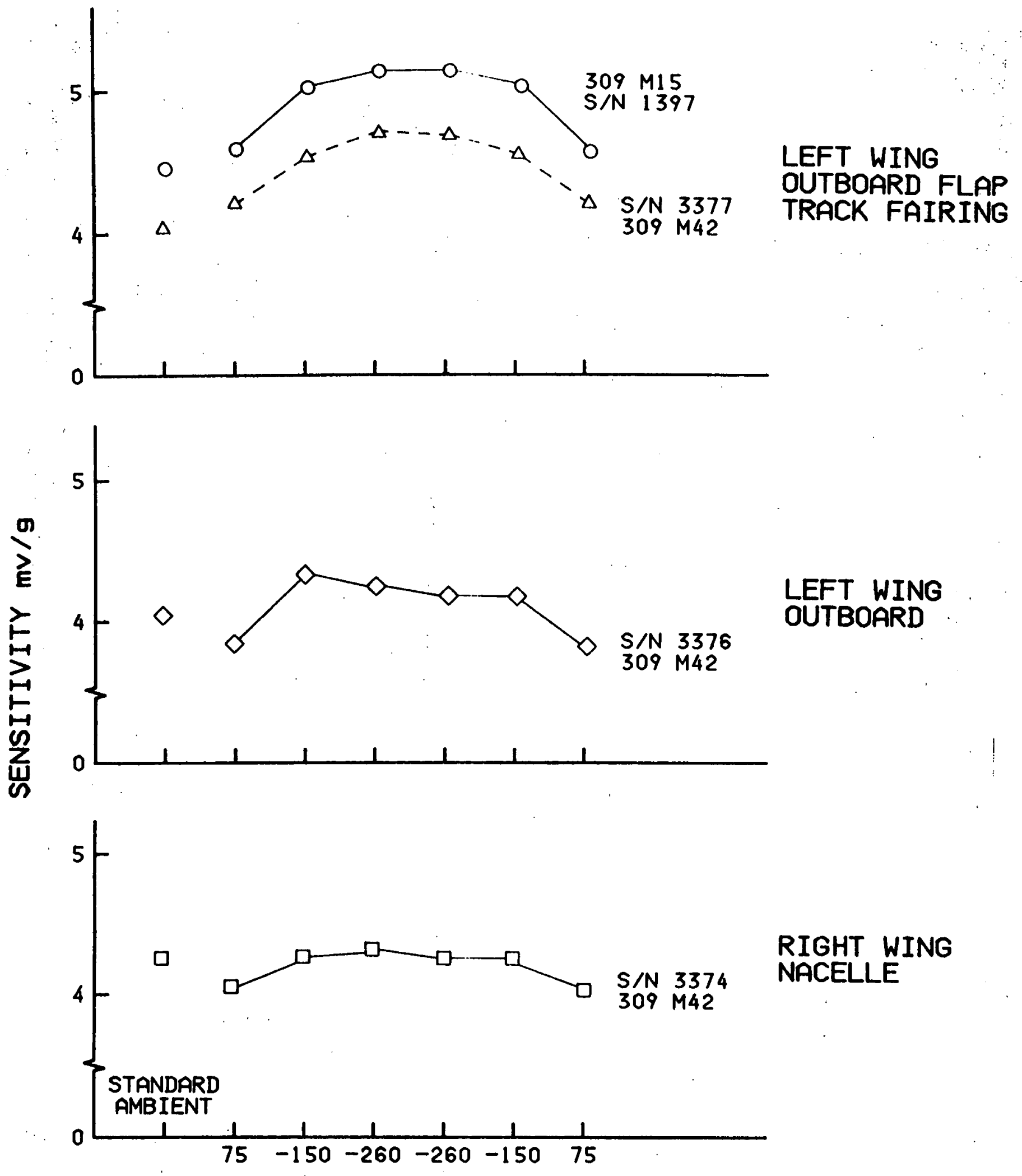

Figure A-2. ACCELEROMETER SENSITIVITIES AT CRYO TEMPERATURES. PCB MODELS 309 M15 \& 309 M42 


\section{Appendix B}

\section{Description of Pre-test Checkout For Dynamic Monitoring and Safety Shutdown Systems}

\section{Background}

This test was conducted to check out the dynamic monitoring and safety shutdown systems that were to be used for conducting the Boeing 767 model buffet test in the NASA Langley National Transonic Facility. The test(s) verified operation of monitoring equipment and instrumentation; and was used to simulate model system dynamic response expected during the actual wind-tunnel test. Also, the systems to be used for automatic emergency shutdown of the facility were functionally checked. These included the Boeing DRAS 1 system and the Langley Critical Point Analyzer (CPA). The Boeing DRAS system was used to guard against overload of the Boeing 767 model structure (excluding the force balance). The Critical Point Analyzer (CPA) was used to protect the balance and support sting swept strut against dynamic overload.

\section{Test Apparatus}

The test setup is given in figure 10. The vibration shaker used was an Electro-Seis, model 113LZ with a 30-pound maximum input force capability and 6 inch stroke. The shaker was attached to a wooden support frame that sat on the test section floor (see figure 12). This set-up provided a semi-rigid base for mounting the vibration exciter (shaker).

\section{Test Procedure}

The test procedure was designed to simulate anticipated model system dynamic response during the actual wind-tunnel test. The shaker was attached to designated hard points on the model and forcing function loads and frequencies established for exciting specified vibration modes.

The test was conducted in the following manner:

1. The shaker was attached to the model at hard points on the right wing outboard flap track fairing and at a forward point on the fuselage centerline. Note: Provision was made for a threaded rod attachment to the outboard flap track fairing. An adapter rod and threaded bolt was used for the fuselage attachment. The shaker was mounted on the wooden support base provided by NTF.

2. All available monitoring system equipment and model instrumentation was active during the forced vibration testing. Vibration test engineers were in voice communication with the test director and/or monitoring equipment operators in the control room, during the test(s).

3. Specified modes were excited to the highest levels possible without exceeding preestablished load or displacement limits established by Boeing and/or NASA. 
4. Modes excited for these tests were:

Symmetric

\begin{tabular}{|c|c|c|}
\hline Mode \# & Mode & Measured Freq., $\mathrm{Hz}$ \\
\hline $\begin{array}{l}1 \\
2 \\
3\end{array}$ & $\begin{array}{l}\text { 1st Sting Bending } \\
\text { Rigid Model Pitching on Balance } \\
\text { 1st Wing Bending }\end{array}$ & $\begin{array}{l}10-11 \\
17 \\
55\end{array}$ \\
\hline \multicolumn{3}{|c|}{ Anti-Symmetric } \\
\hline $\begin{array}{l}1 \\
2 \\
3\end{array}$ & $\begin{array}{l}\text { Rigid Model Rolling and Yawing on Balance } \\
\text { Rigid Model Rolling on Balance } \\
\text { First Wing Bending }\end{array}$ & $\begin{array}{l}19 \\
23 \\
77\end{array}$ \\
\hline
\end{tabular}

Primary emphasis was placed on exciting the 19 and $23 \mathrm{~Hz}$ roll modes (anti-symmetric) to approximately 1 inch peak-to-peak at the wing tip. Wing bending modes are also excited but at very low levels.

5. During the forced vibration testing, the model system instrumentation output was recorded on the NASA 14-channel tape recorder, and monitored real-time by Langley personnel. The following monitoring equipment was used:

Boeing

- DRAS Unit No. 1

NASA

$-\mathrm{BDDU}$

- CPA

- TDT O-Graph \& FFT Analyzer

- NTF FFT Analyzer

6. The forced vibration response test accomplished the following objectives.

A. Verified monitoring equipment and instrumentation operation.

B. Correlated measured response with

1) Input Forcing Function

2) Balance (BDDU response) with accelerometer/strain gage(s) response on wings and support sting. (Note: The results of this study could have had a bearing on decisions related to continuation of testing if one or more accelerometers had been permanently lost during the wind-tunnel test.) 
C. The forced vibration testing was used to check the function of the model safety and shutdown systems. This was done by setting trip levels (and frequencies as appropriate) on the DRAS 1 unit and the CPA that were detected from the forced vibration loads. Shutdown commands to appropriate visual and/or audible alarms were verified, and tunnel interlocks signal transmission verified.

D. Data taken from the BDDU yaw moment dynamic response were used to characterize the dynamic signal response and establish the $\%$ full scale value at which fouling occurred between the fuselage and swept strut when forcing the model at $\sim 20 \mathrm{~Hz}$ in roll. 


\section{Appendix C \\ Safety/Risk Assessment Model Used For Boeing 767 Buffet Test in NTF}




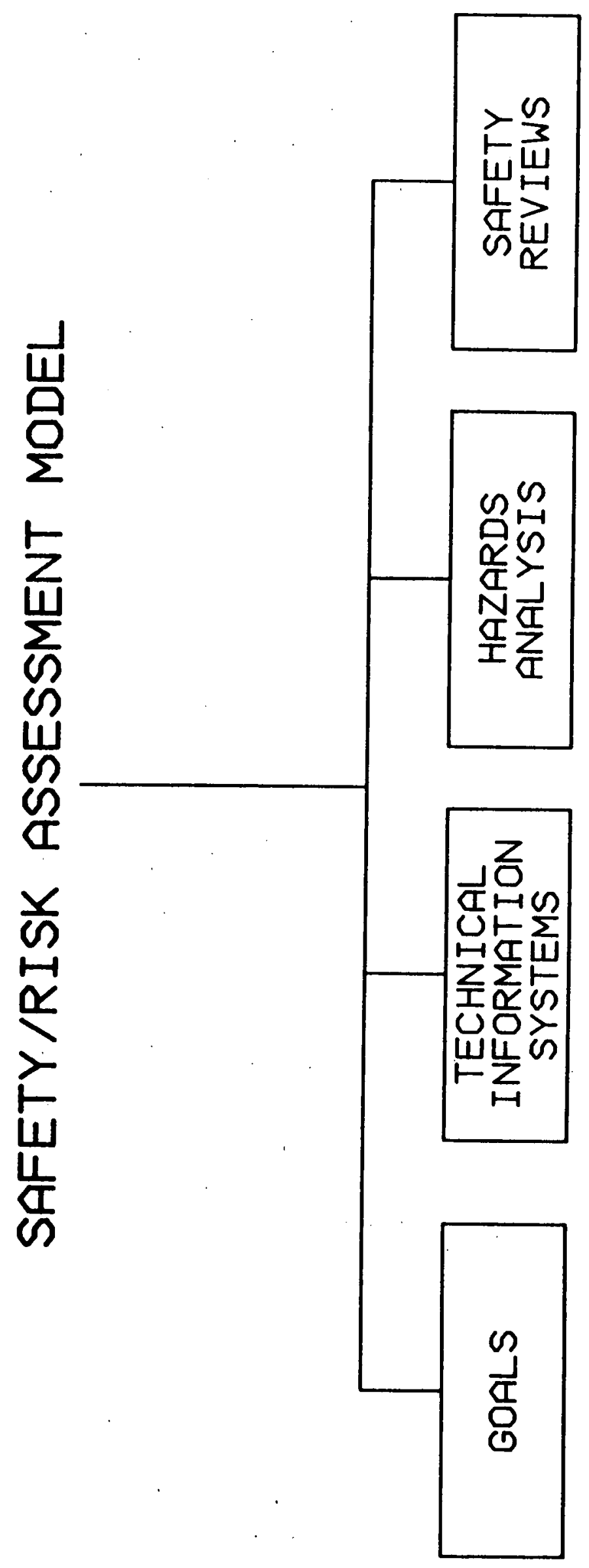




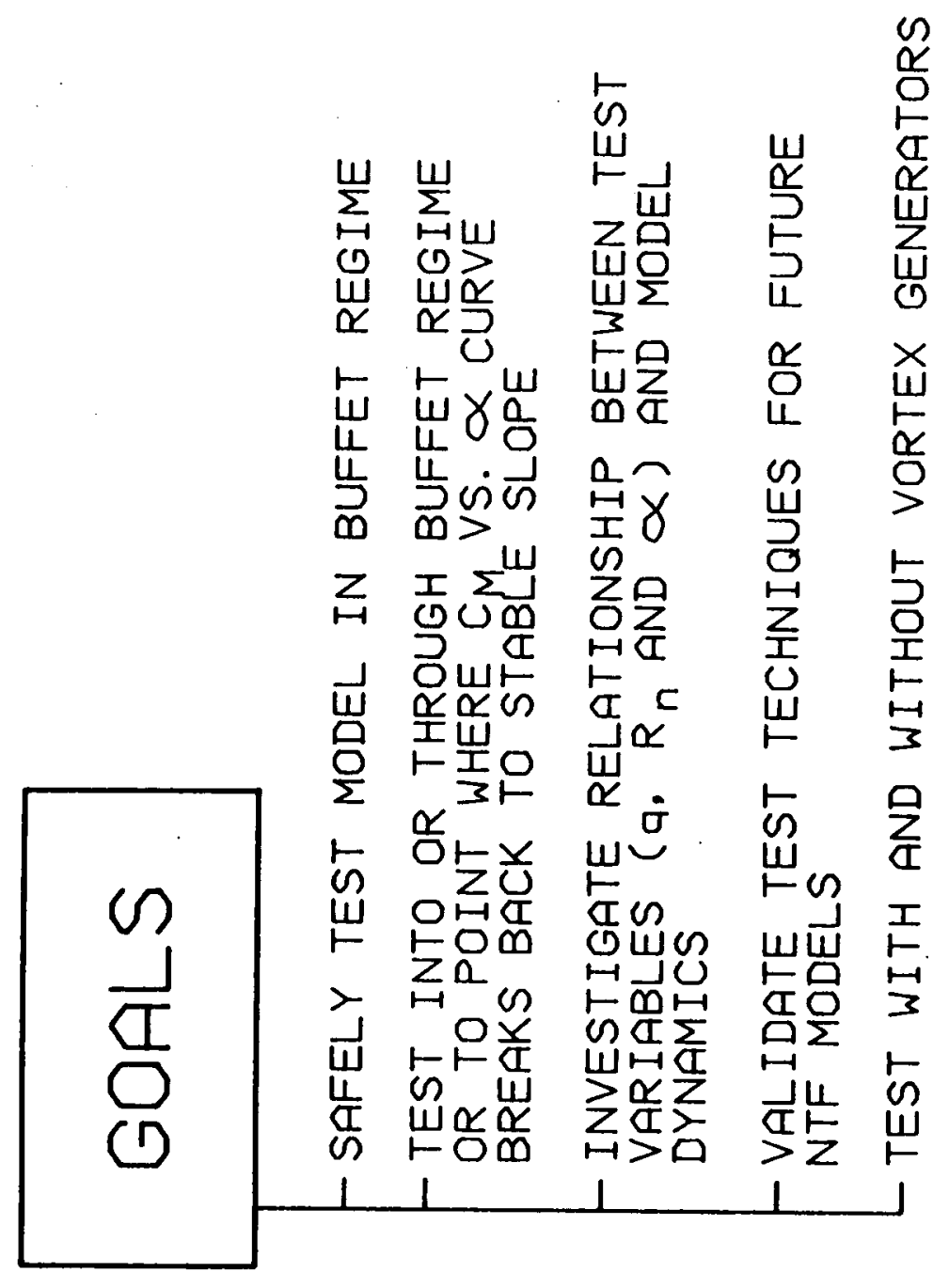




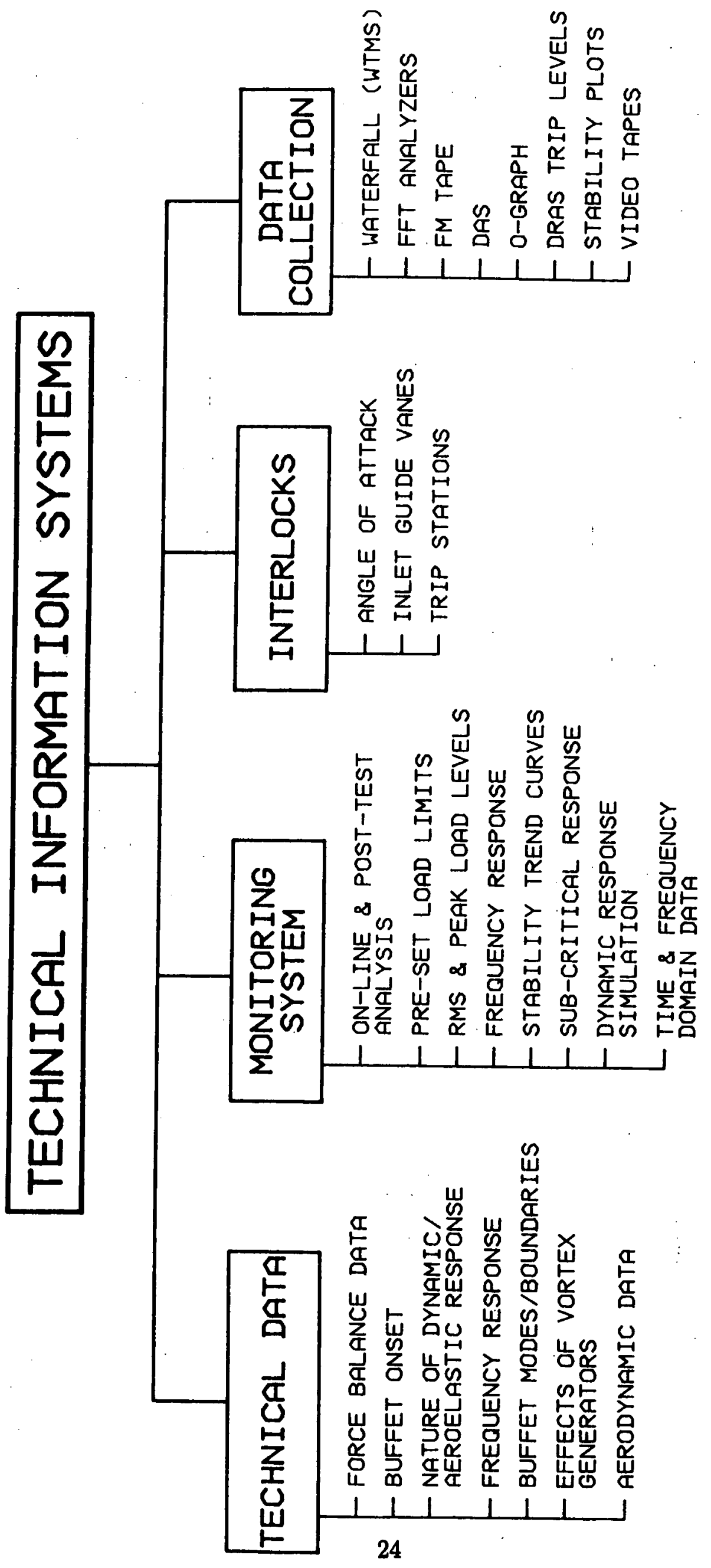




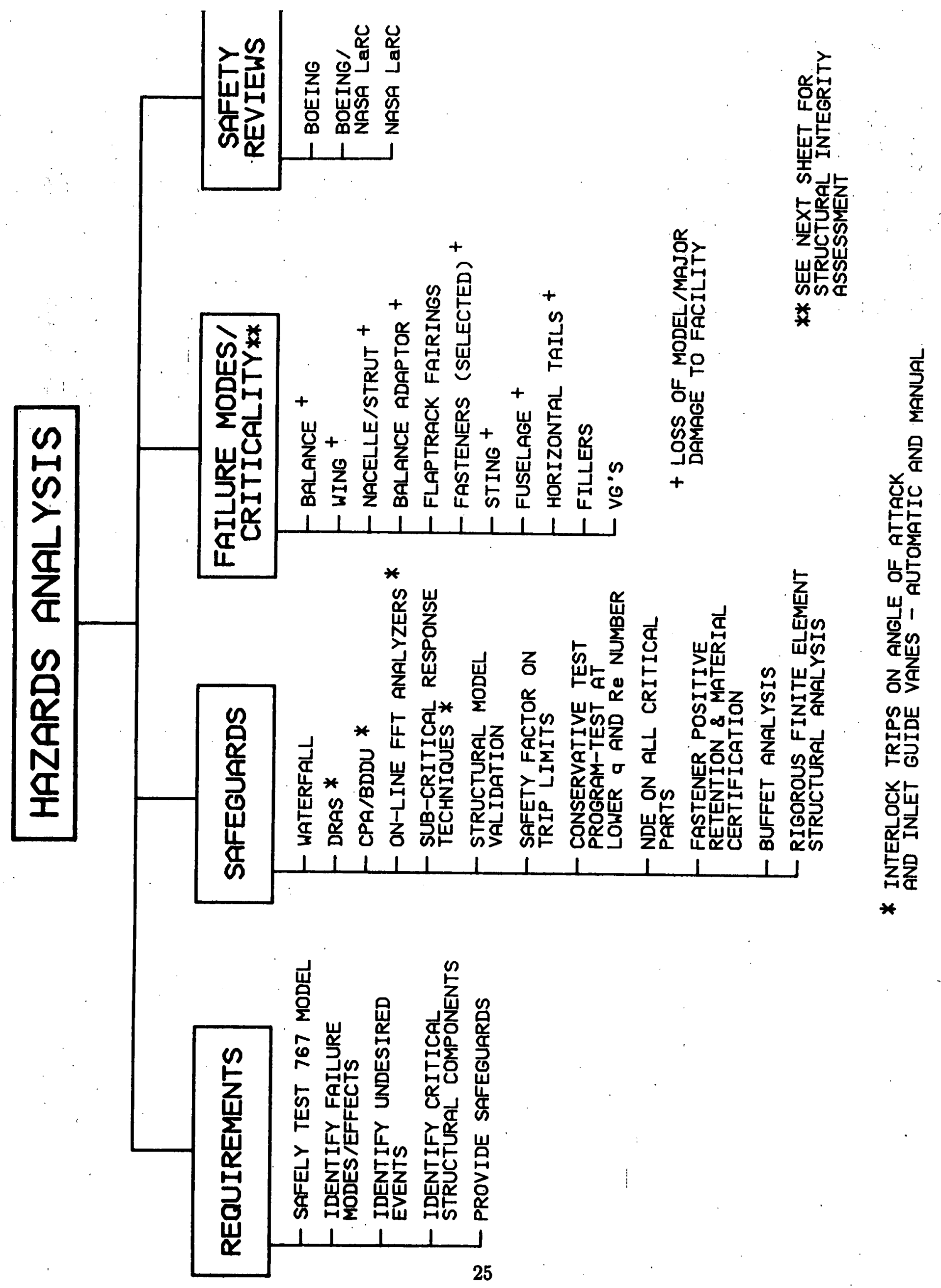




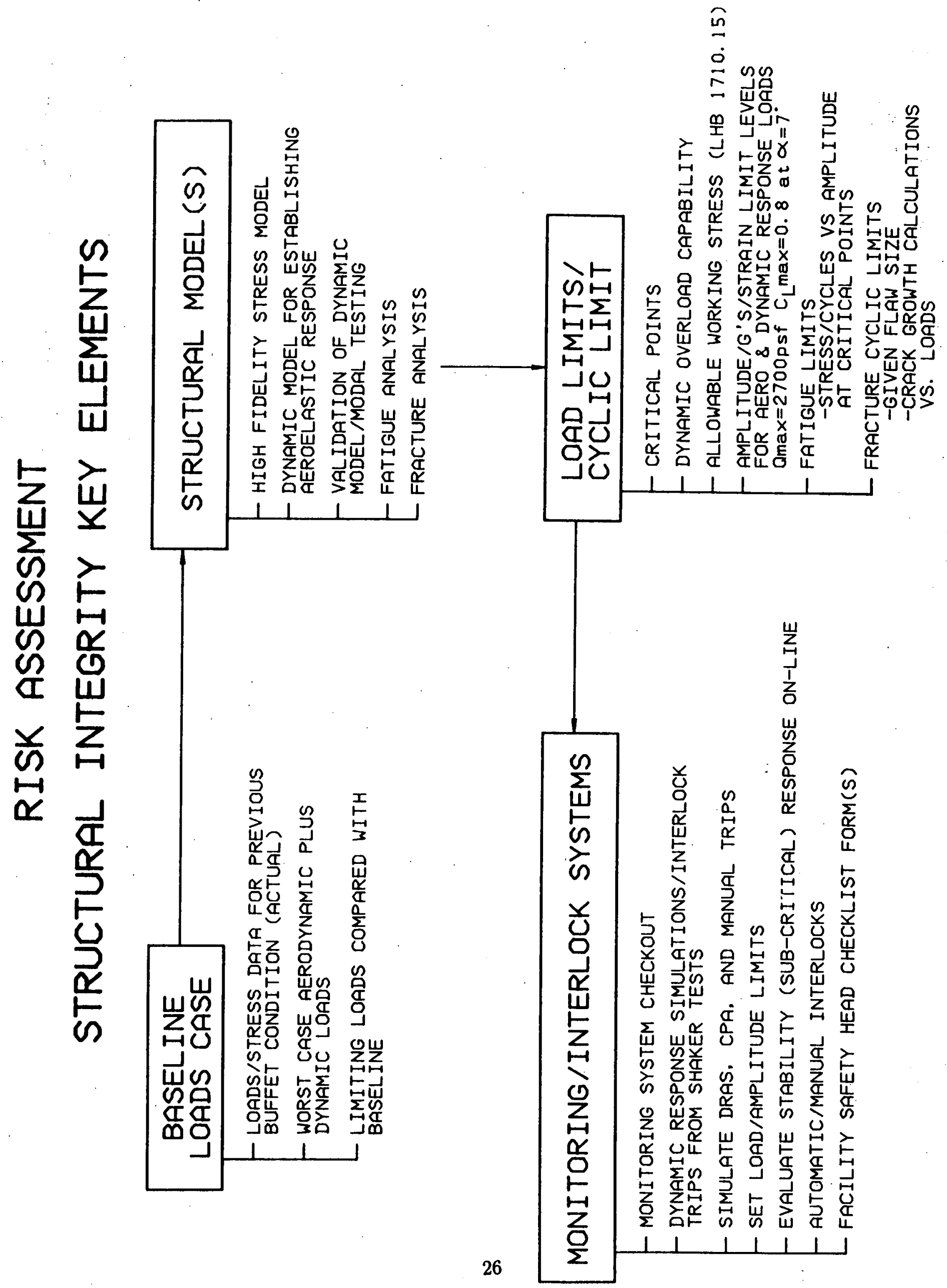




\section{Appendix D}

Special Procedure For Model Protection

Interlock Variable Inputs 
Special Procedure

Model Protection Interlock Variable Inputs

Boeing 767 Test \#038

Purpose: The purpose of this procedure is to provide a series of checklist items that will be approved by the Facility Safety Head prior to operating the tunnel. The checklist items are for the safe management of instrumentation interlock variable inputs that are critical to protecting the structural integrity of the test model and the model support system. Prior to each new run where a variable (limit) change is required or to reset system after an interlock trip, the following checklist will be used. The test director will be responsible for tracking variable input changes throughout the test program and interlock trip conditions.

Objective: The objective of this procedure is to ensure that interlock limits defined as variable inputs are properly maintained and correctly entered into the proper instrumentation device(s) figure 1. The instrumentation devices are the critical components for sensing (automatic/visual) model buffeting boundaries and initiating the appropriate interlock actions. Incorrect variable input entries may result in model/support system failure and tunnel damage as identified in the NTF/Boeing 767 safety Analysis Review.

Scope: Prior to running a selected test condition, (Mach Number, Reynolds Number $\left\{R_{e}\right\}$ and Dynamic Pressure $\left.\{Q\}\right)$, the test director will review the need to change interlock variables and/or verify the current variable inputs (no changes from the last checklist sign-off). Any discrepancies or input changes are to be documented and/or changed via this special procedure.

Seven (7) stations have been identified as noted on Figure 2 . Each station will have an assigned person who is responsible for equipment operation, calibration and variable (limit) input. Each assigned person(s) will sign off the indicated items on this checklist.

Procedure Approved: Rev. A

FSH :

FC: 
Model Protection Interlock Checklist

Date:

Variable Inputs

Time:

Test Condition

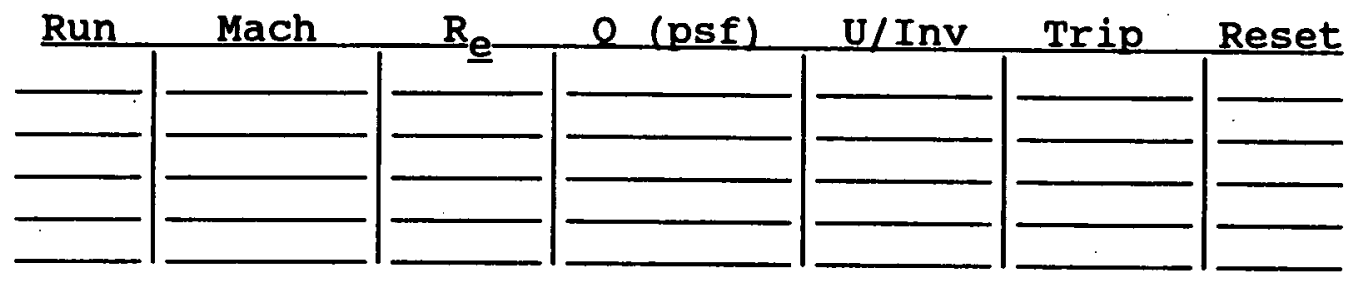

BTATION 1: Assigned to:

INITIAL

1.1 DRAS \#1

Channel 1 Channel 2

NASA/Boeing

Trip Level Millivolts Pk

Maximun Freq-HZ

Flutter Freq-Hz

No. Events

Relay Enable

Power

1.2 DRAS \#2

Channel 1 Channel 2

Trip Level Millivolts Pk

Maximun Freq-Hz

Flutter Freq-Hz

No. Events

Interlocks checked

Power

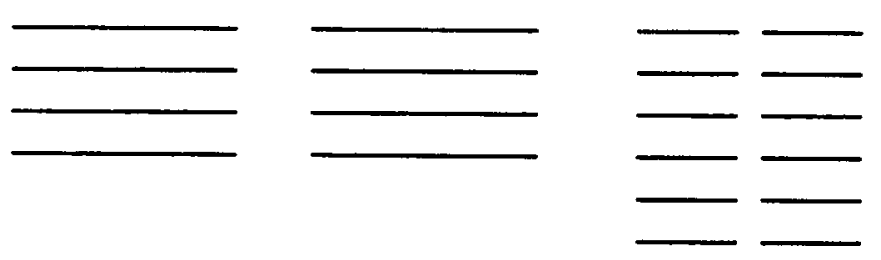

1.3 WATERFALL

1.4 WATERFALL (Slave)

8TATION 2: Assigned to

INITIAT

2.1 FFT

wing

strut

Balance

2.2 FFT (A11 Channels)

BTATION 3: Assigned to

3. TV Monitors 
Model Protection Interlock Checklist

Variable Inputs

BTATION 4: Assigned to

INITIAL

4.1 Tape Recorder

4.2 Power Supplies

4.3 Gain Settings/Voice Record

4.4 Technician Available

BTATION 5: CPA Assigned to

5.1

Normal Axial Pitch Roll Yaw Side

\begin{tabular}{|c|c|c|c|c|c|c|}
\hline Ch1 & 08 & -13 & -14 & -17 & -35 & 14 \\
\hline $\mathrm{Ch} 2$ & -38 & -00 & -42 & -00 & -09 & 10 \\
\hline $\mathrm{Ch} 3$ & -51 & 00 & 24 & -00 & -18 & -07 \\
\hline Ch4 & -47 & -00 & -25 & -08 & -07 & -14 \\
\hline Ch 5 & -58 & -00 & -07 & -03 & -03 & 29 \\
\hline $\mathrm{Ch} 6$ & -00 & & 00 & & 14 & 67 \\
\hline
\end{tabular}

5.2 Power - on

5.3 Mode - Scan

5.4 Dynamic Interlock - Checked lon

BTATION 6: BDDU Assigned to

6.1 Set Up per RIOP-20

6.2 Ocilliscope (Yaw Moment)

for Monitoring Model Fouling ( $+20 \%=100 \%$ ALARM)

STATION 7: Test Director Console

CAUTION

Ensure correct sign is entered

for "Pitch Home" setpoint.

7.1 Pitch Home setpoint J/Inv

7.2 Pitch - Armed

7.3 Reset Model Protection

7.4 Kodel Roll e 0 Deg. \& Red Tagged

Test Director/Date

(F8H)
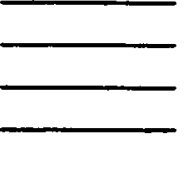
Comments/Notes: 


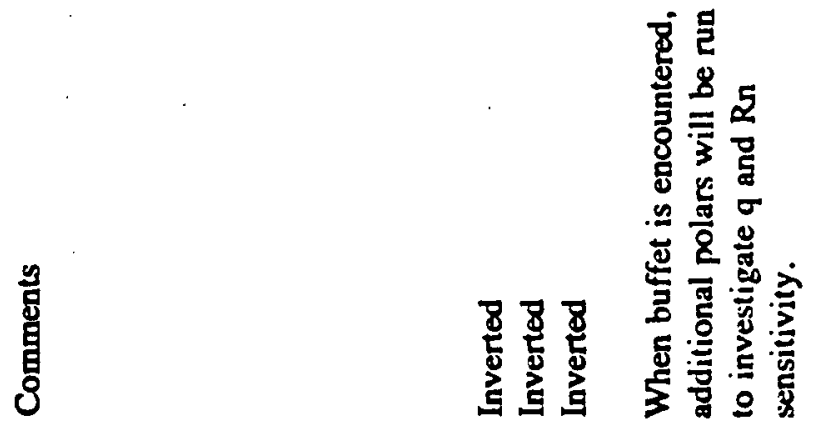

$\widehat{0}$

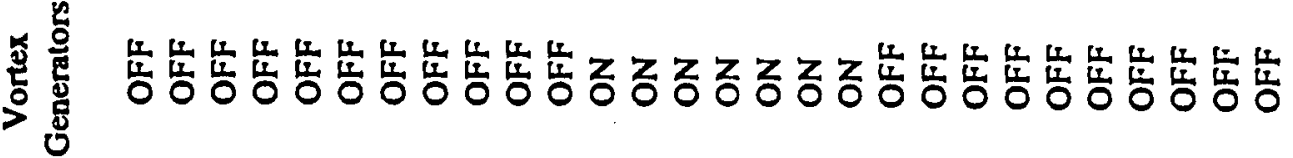

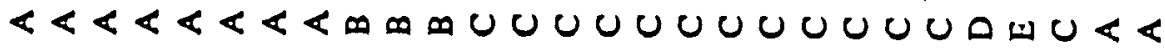

$\S$

点

号

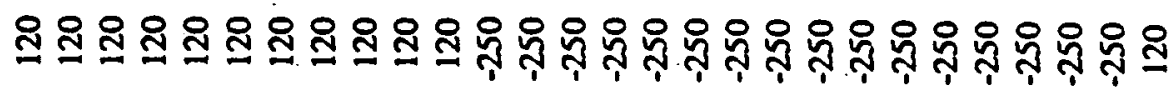

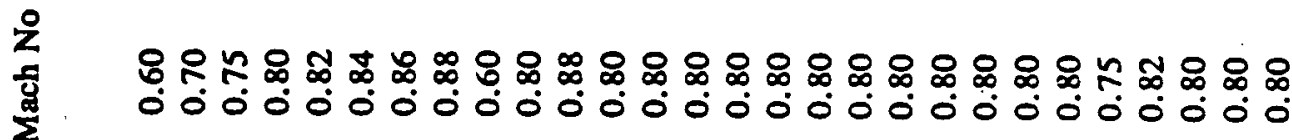

㤀

遌

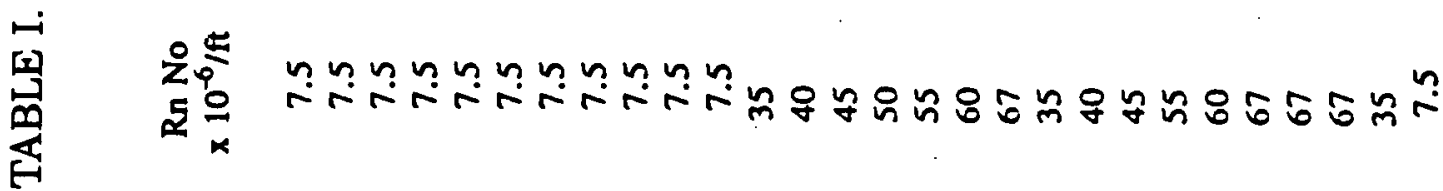

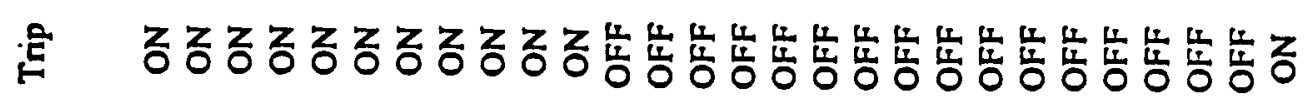

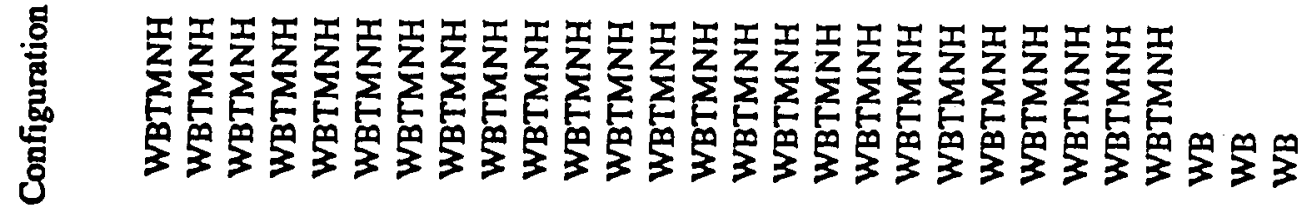

กั

药 - -

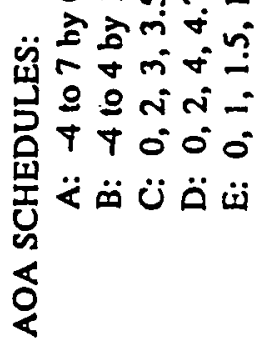




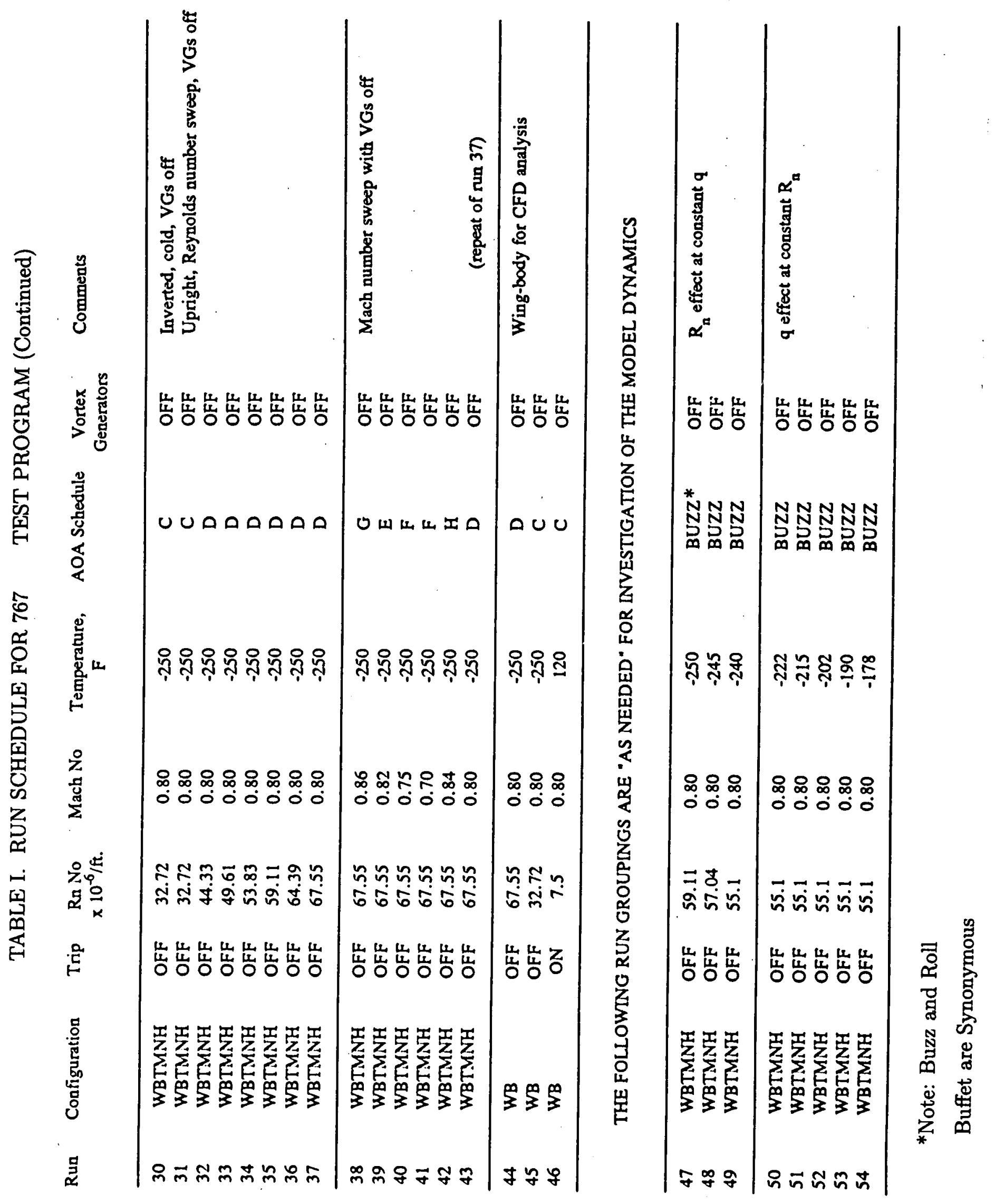


TABLE II. SUMMARY OF MEASURED MODEL SYSTEM PRIMARY STRUCTURAL MODES AND FREQUENCIES

Mode Description

Sting Bending (Yaw Plane)

Sting Bending (Pitch Plane)

Pitch Motion (Balance)

Roll/Yaw (Balance)

Roll

Wing Bending (1st Mode Sym.)

Wing Bending (1st Mode Asym.)
Frequency, $\mathrm{Hz}$

5.8
11
17
19
23
55
77

TABLE III. CRITICAL LOADING/

FAILURE LOCATIONS FOR VARIOUS VIBRATION MODES

Mode

$20 \mathrm{~Hz}$ Roll

$55 \mathrm{~Hz}$ Bending 1st Wing (Sym) 1st Wing (Asym)

Loose Fasteners

Other Vibrations
Critical Area

Wing @ Sta 9.30 Aft Fastener Hole
Wing @ Sta 9.30 Aft Fastener Hole
Wing @ Sta 9.30 Aft Fastener Hole
Wing @ Sta 9.30 Aft Fastener Hole
Wing, Horizontal, Nacelles
Upper Swept Strut Blade

TABLE IV. WING STRESS CALCULATIONS (NORMALIZED)

\begin{tabular}{|c|c|c|c|}
\hline Loading Mode & $\begin{array}{c}\text { Stress @ } \\
\text { Nacelle Hole }\end{array}$ & $\begin{array}{c}\text { Maximum } \\
\text { Stress }\end{array}$ & $\begin{array}{c}\text { Wing Tip } \\
\text { Deflection } 100 \mathrm{G}\end{array}$ \\
\hline Air (2700 Q) & 30555 (73332) & 45343 & N/A \\
\hline Air + $100 \mathrm{Gs} @ 20 \mathrm{~Hz}$ & $42000(108000)$ & 67553 & $5.5^{\prime \prime}$ \\
\hline $\mathrm{Air}+100 \mathrm{Gs} @ 50 \mathrm{~Hz}$ & 50555 (121332) & 81887 & $.88^{\prime \prime}$ \\
\hline $\mathrm{Air}+100 \mathrm{Gs} @ 84 \mathrm{~Hz}$ & $36050(86520)$ & 61545 & $.32^{\prime \prime}$ \\
\hline Air + $100 \mathrm{Gs} @ 86 \mathrm{~Hz}$ & 35120 (84288) & 56645 & $.31^{\prime \prime}$ \\
\hline
\end{tabular}


TABLE V. SUMMARY OF CRITICAL MODEL COMPONENTS, FACTORS OF SAFETY, AND FAILURE ACCEPTABILITY FOR $20 \mathrm{~Hz}$ ROLL MODE - 100 g's AT WING TIP

\begin{tabular}{|c|c|c|c|c|}
\hline Component & Static & ynamic St & s Level/F.S. & $\begin{array}{c}\text { Failure } \\
\text { Acceptable? }\end{array}$ \\
\hline $\begin{array}{l}\text { Wing } \\
\text { Horizontal Tail } \\
\text { Nacelle \& Strut } \\
\text { Sting (Blade) } \\
\text { Balance Adaptor } \\
\text { Body } \\
\text { Flap Track Fairing Assy } \\
\text { Wing Bolts } \\
\text { Nacelle Bolts } \\
\text { Horizontal Bolts } \\
\text { Vortex Generators } \\
\text { Balance }\end{array}$ & $\begin{array}{c}73332 \\
79688 \\
17310 \\
50097 \\
97328 \\
10703 \\
<1200 \\
1429 \mathrm{LBF} \\
152 \mathrm{LBF} \\
1476 \mathrm{LBF} \\
\text { Low } \\
31462 \mathrm{In}-\mathrm{lb}\end{array}$ & $\begin{array}{c}108000 \\
97089 \\
28043 \\
71305 \\
97330 \\
18524 \\
12000 \\
3261 \mathrm{LBF} \\
246 \mathrm{LBF} \\
3228 \mathrm{LBF}\end{array}$ & $\begin{array}{c}/ 2.38 \\
/ 2.60 \\
/ 4.85 \\
/ 3.50 \\
/ 2.60 \\
/ 13.5 \\
/ 20.8 \\
/ 2.7 \\
/ 3.35 \\
/ 2.38 \\
\text { Low } \\
350 \% \text { Overload }\end{array}$ & $\begin{array}{l}\text { No } \\
\text { No } \\
\text { No } \\
\text { No } \\
\text { No } \\
\text { No } \\
\text { Yes } \\
\text { No } \\
\text { No } \\
\text { No } \\
\text { Yes } \\
\text { No }\end{array}$ \\
\hline
\end{tabular}




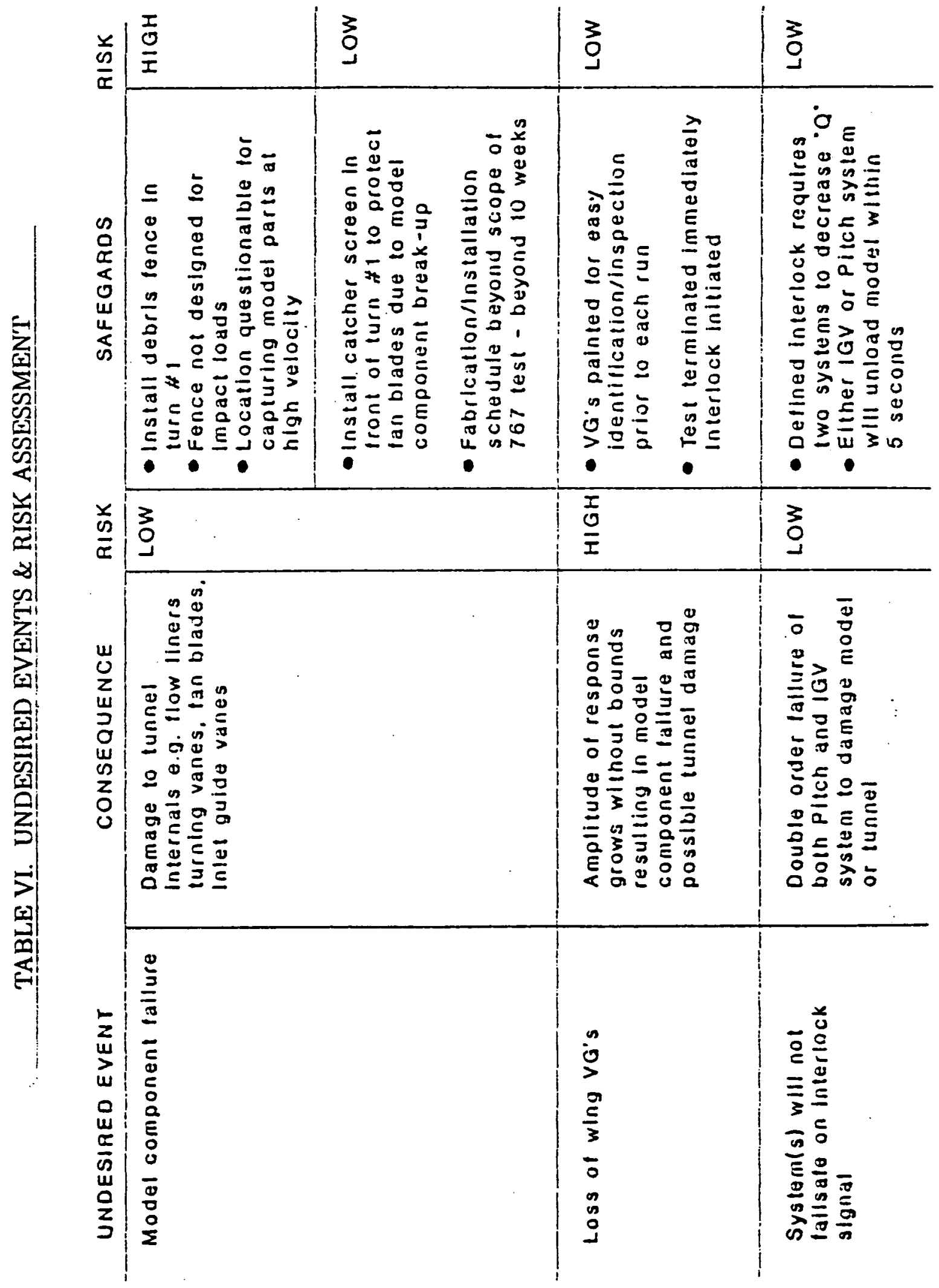




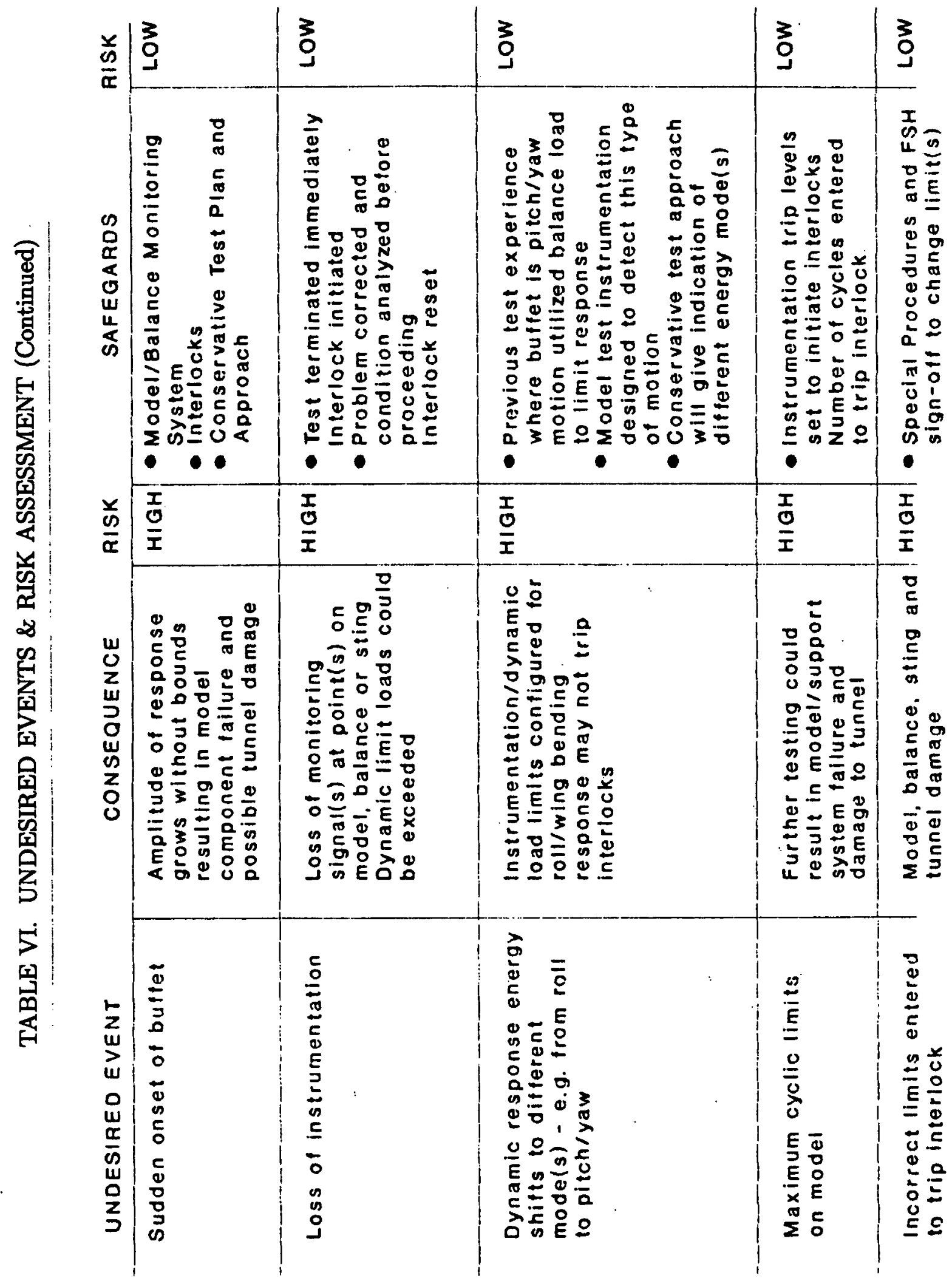




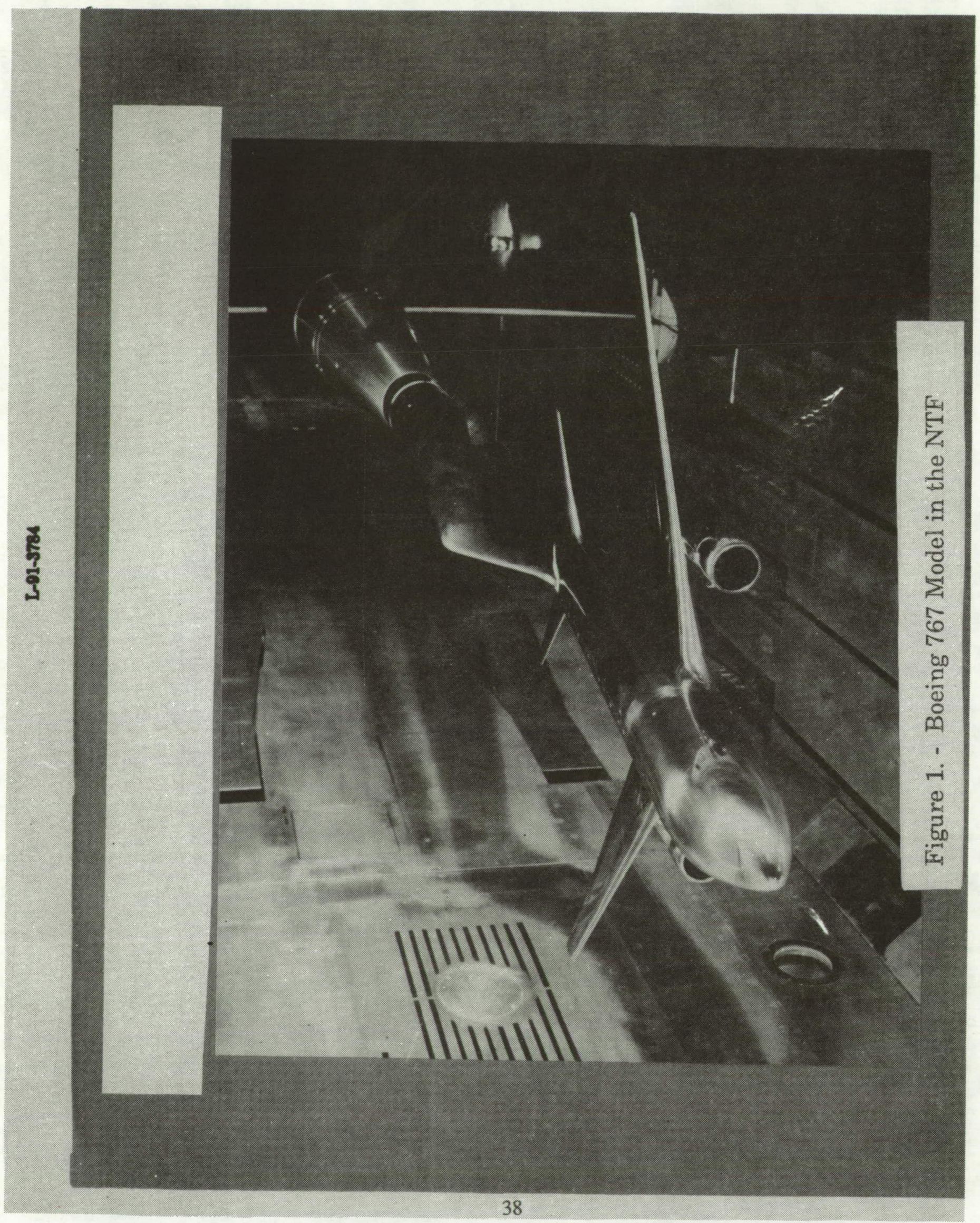

ORIGINAL PAGE

BLACK AND WHITE PHOTOGRATH 


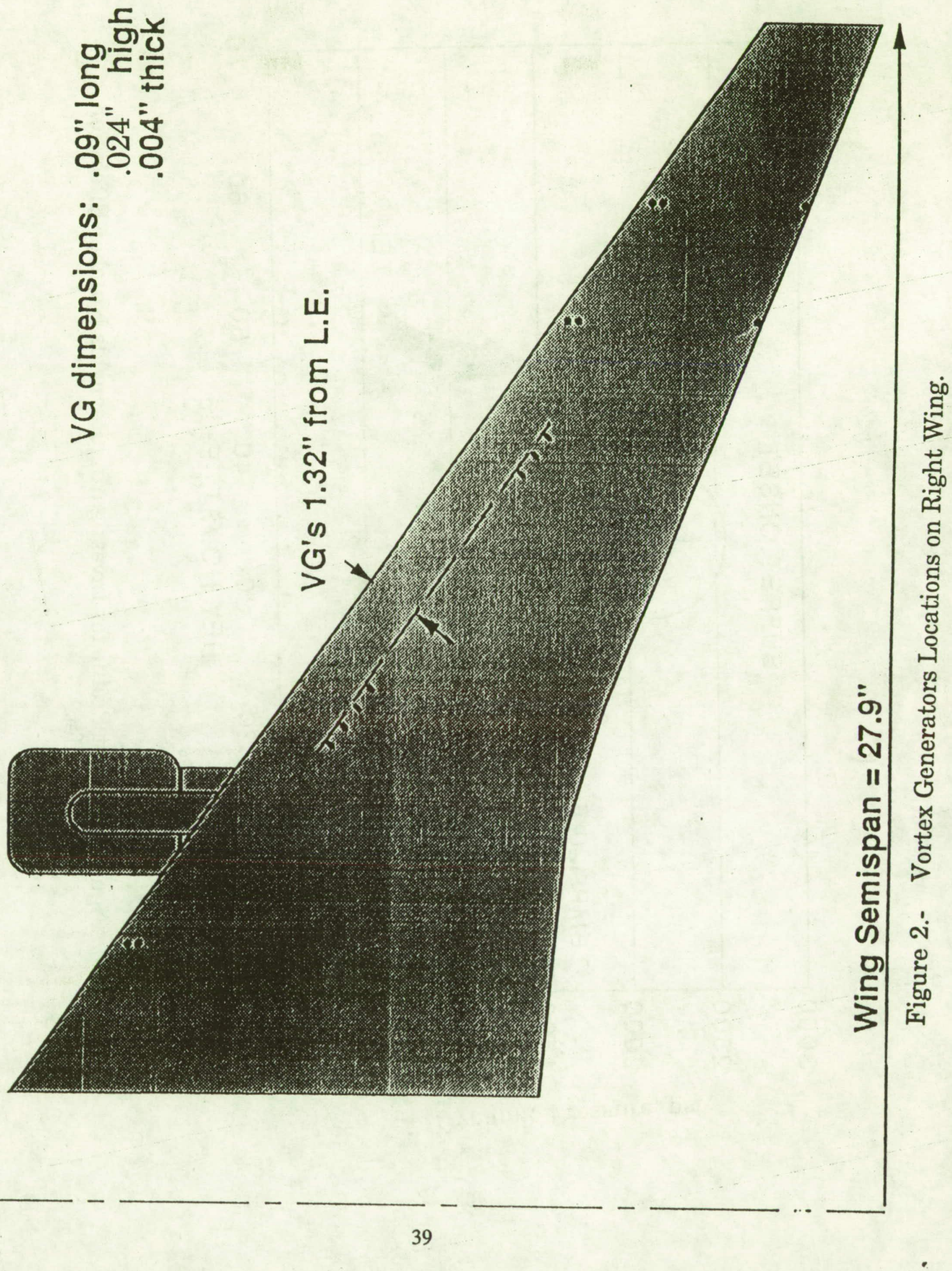




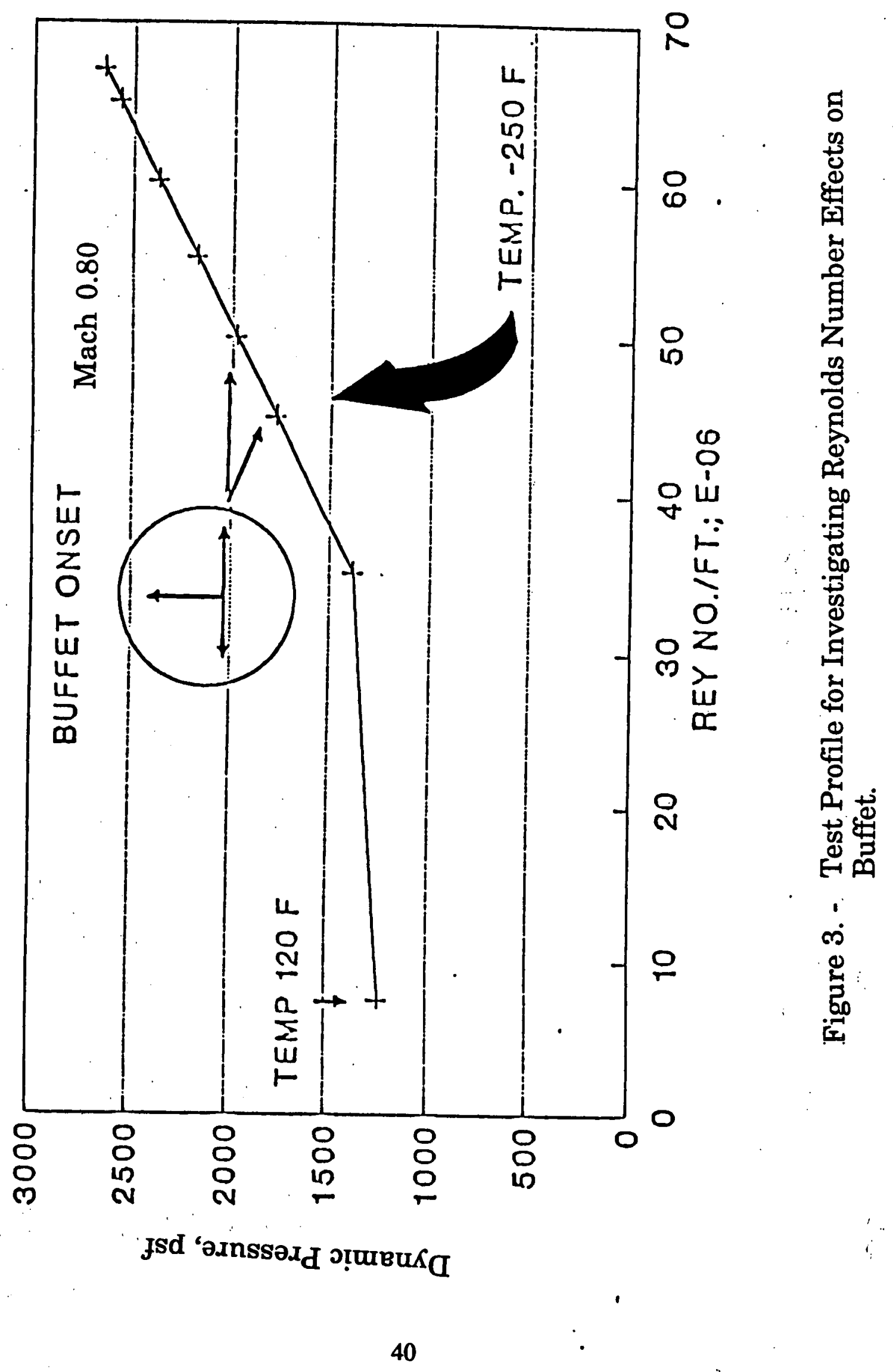




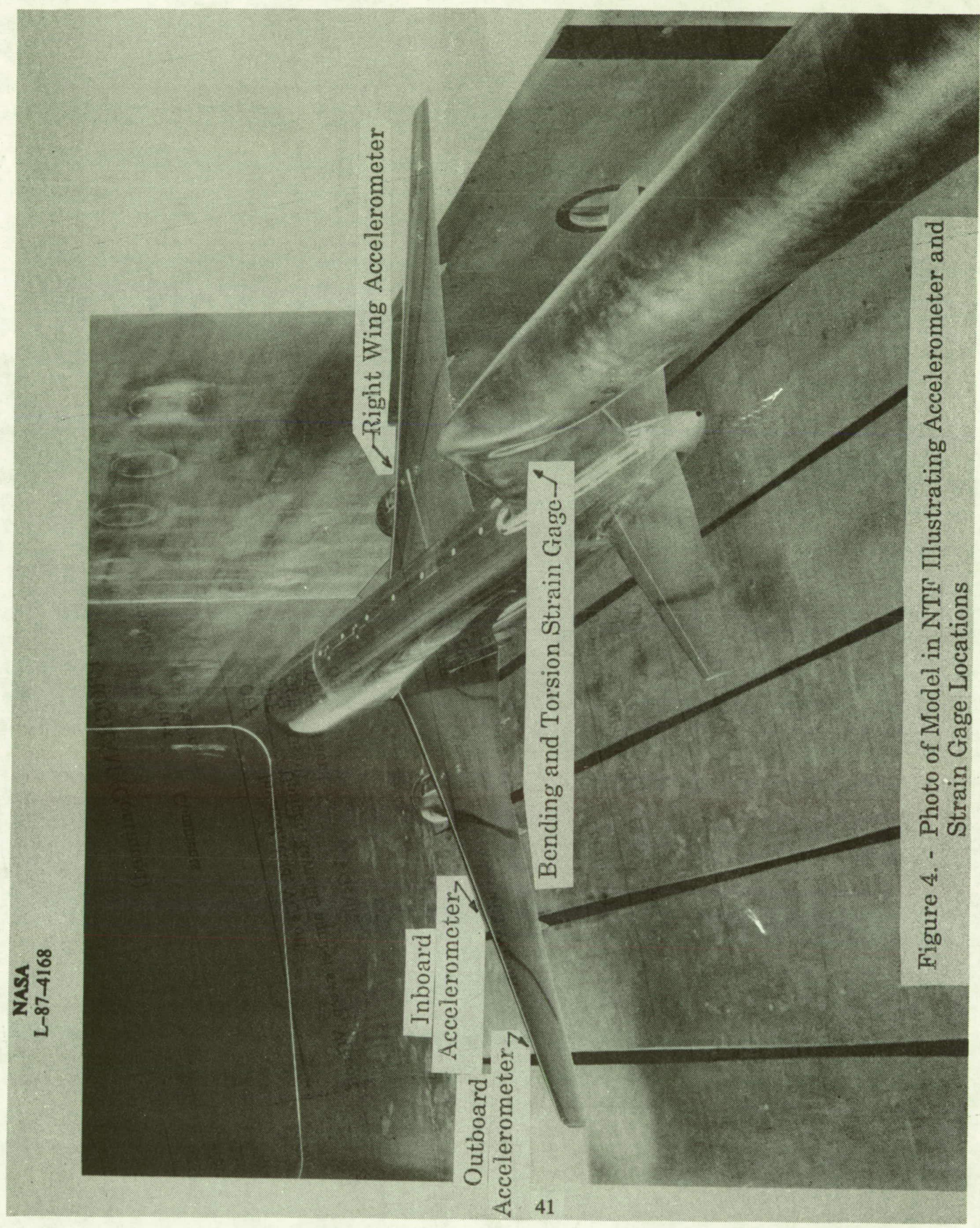

ORIGINAL PAGE

BLACK AND WHITE PHOTOGRAPH 


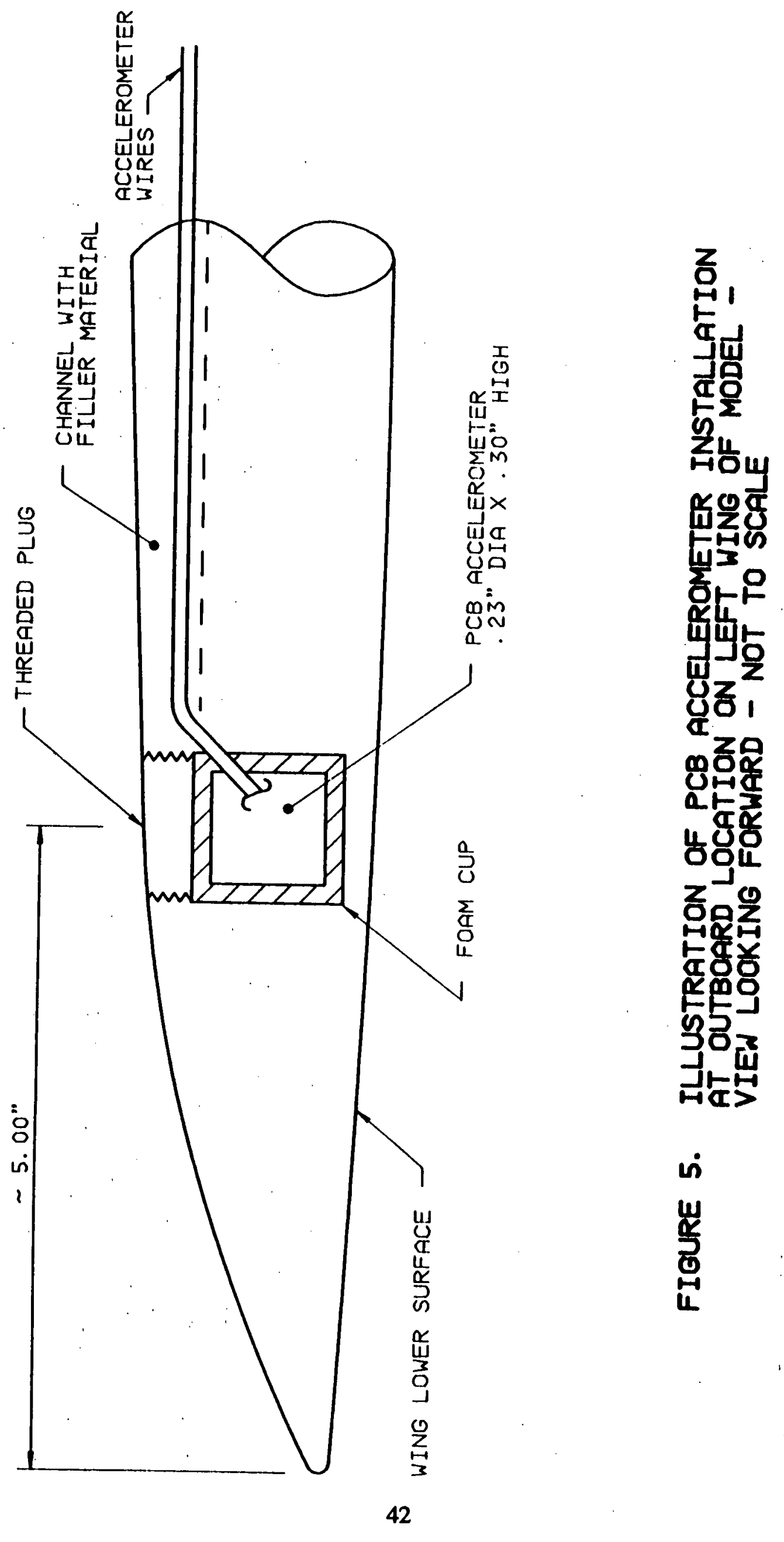




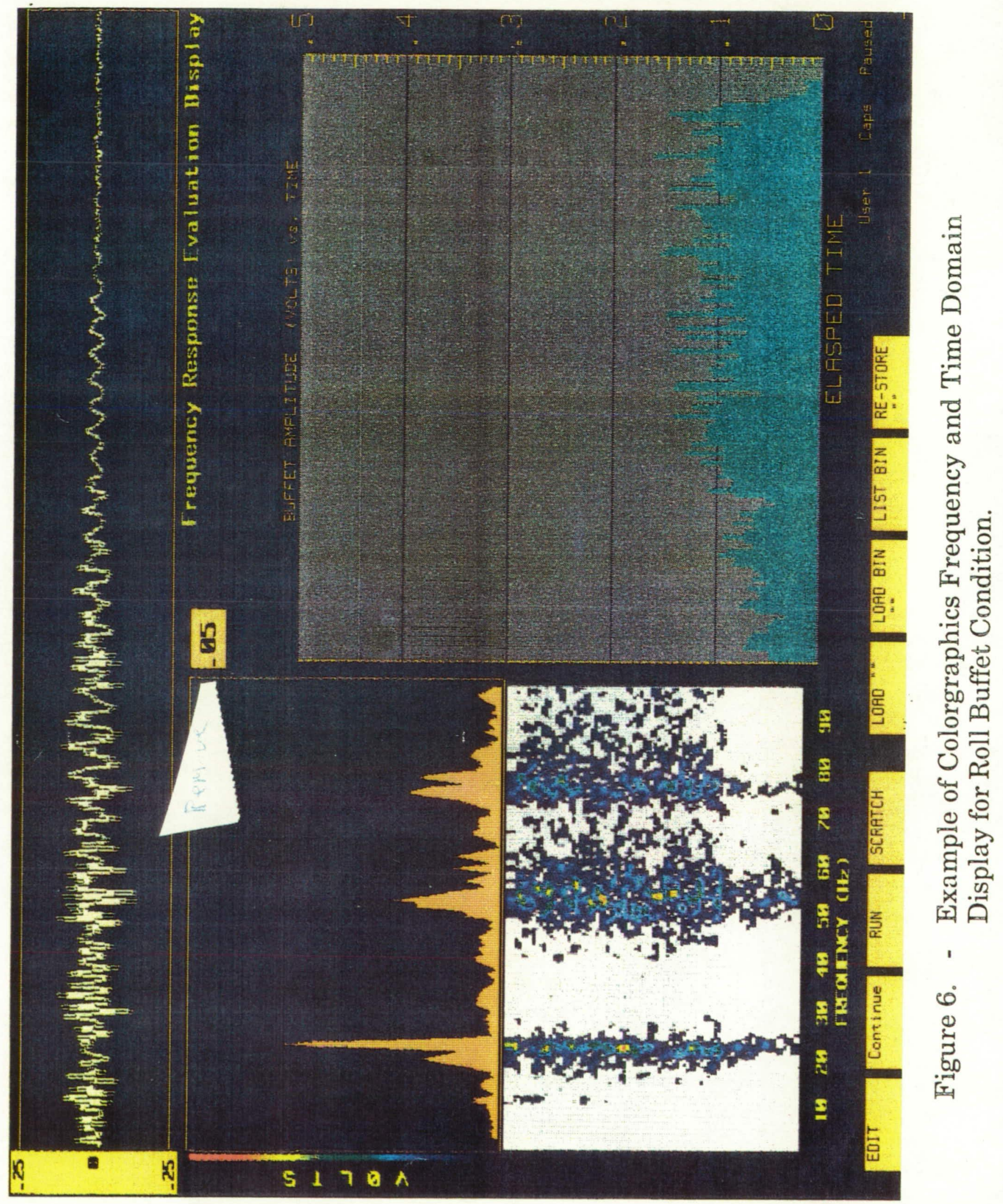




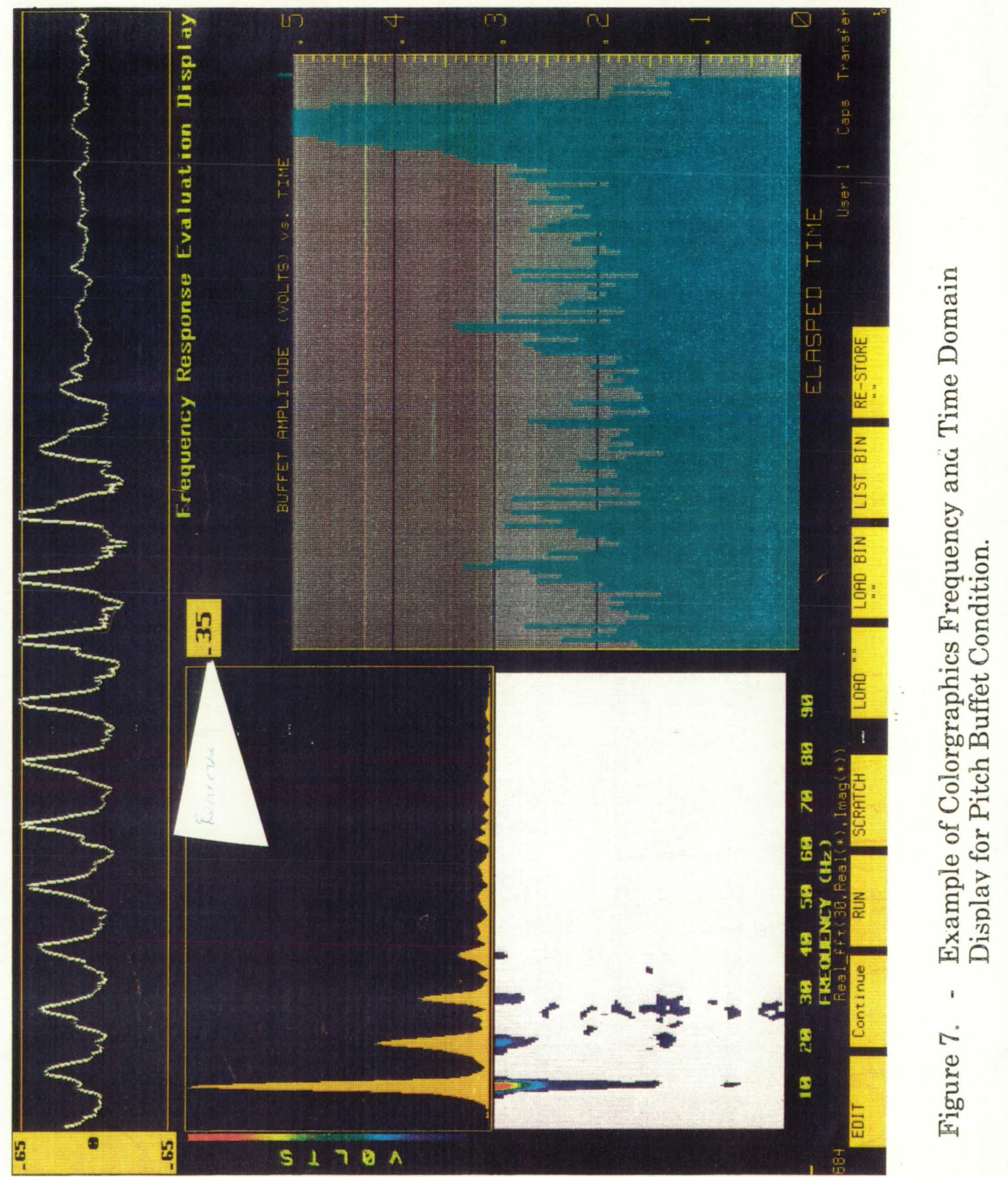




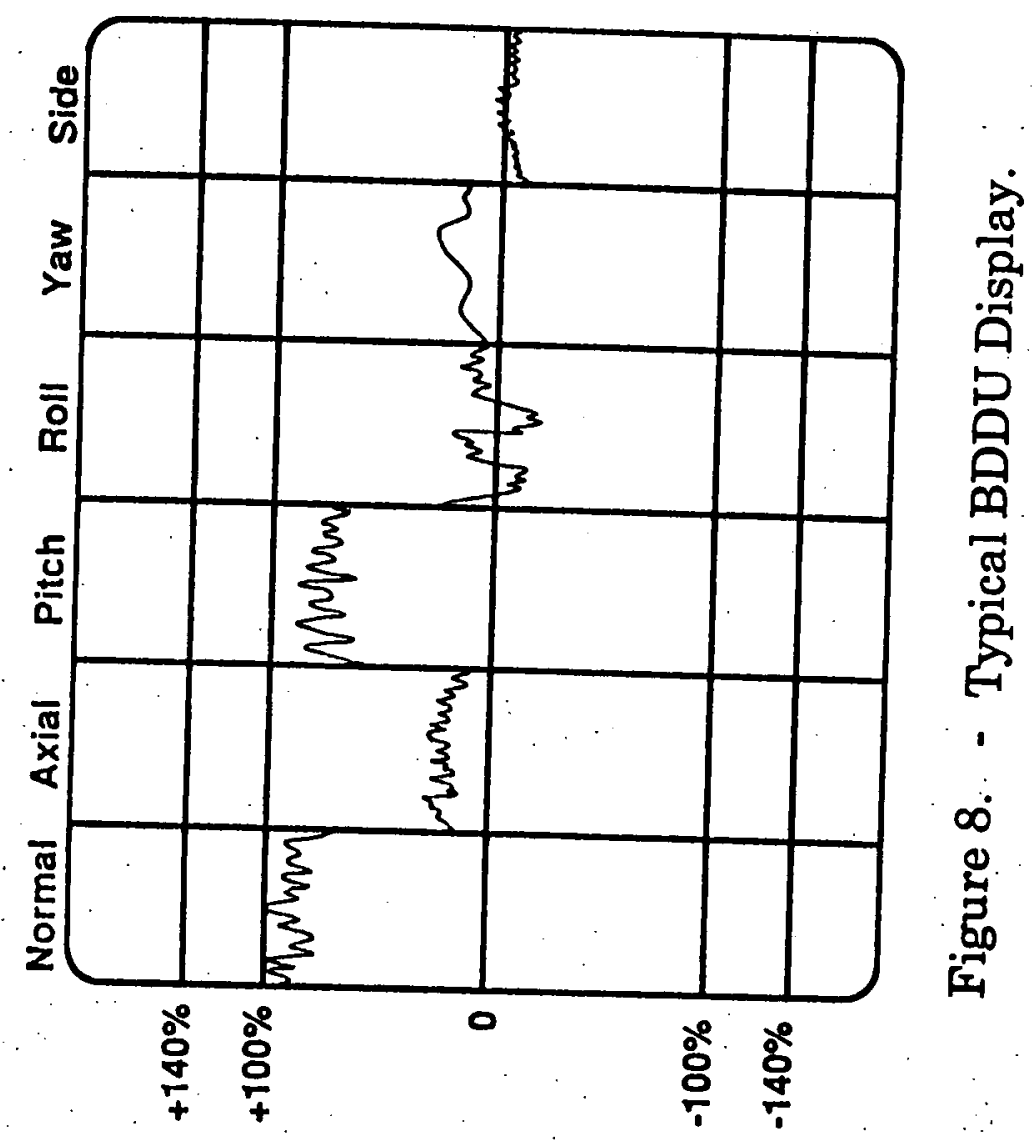




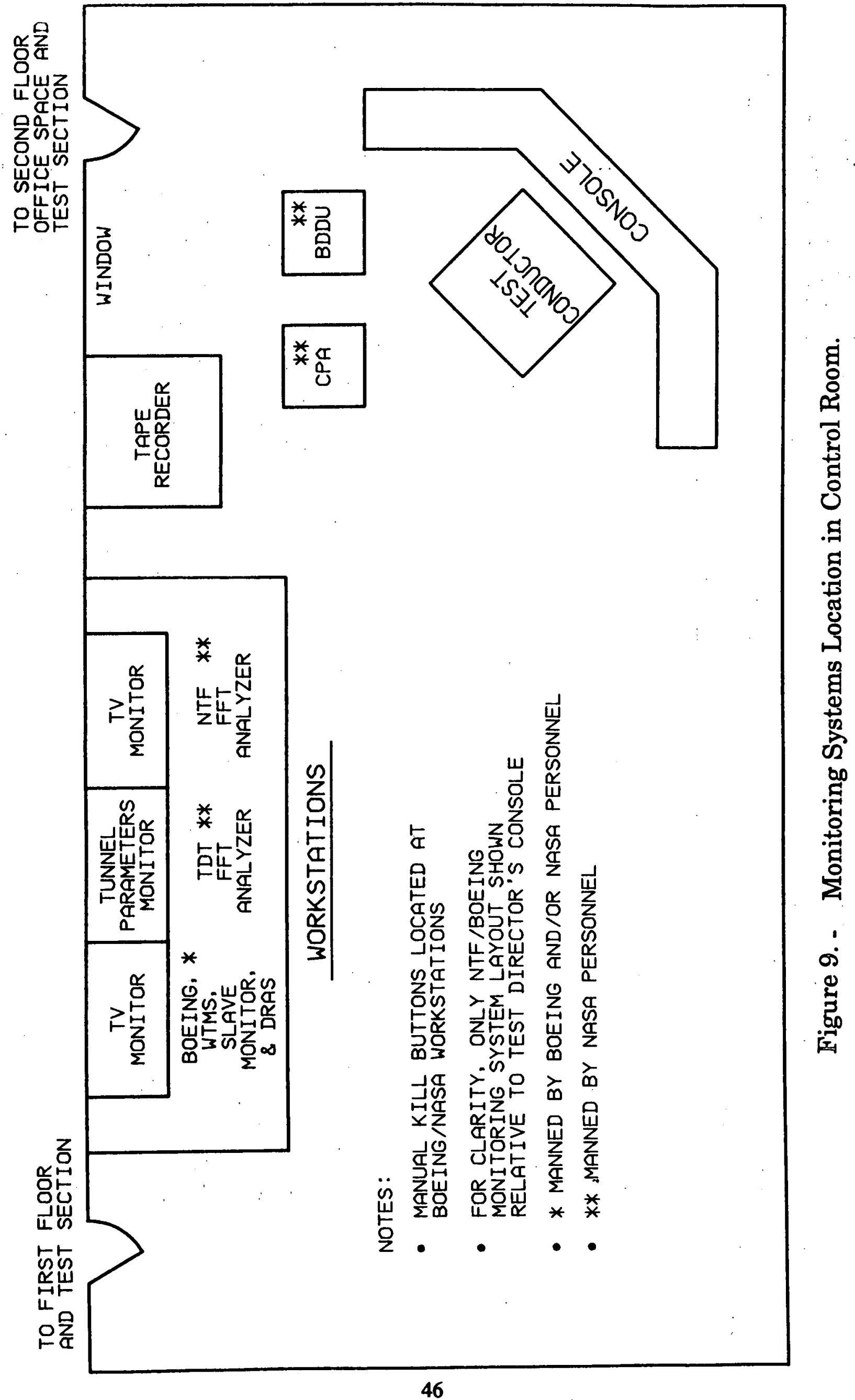




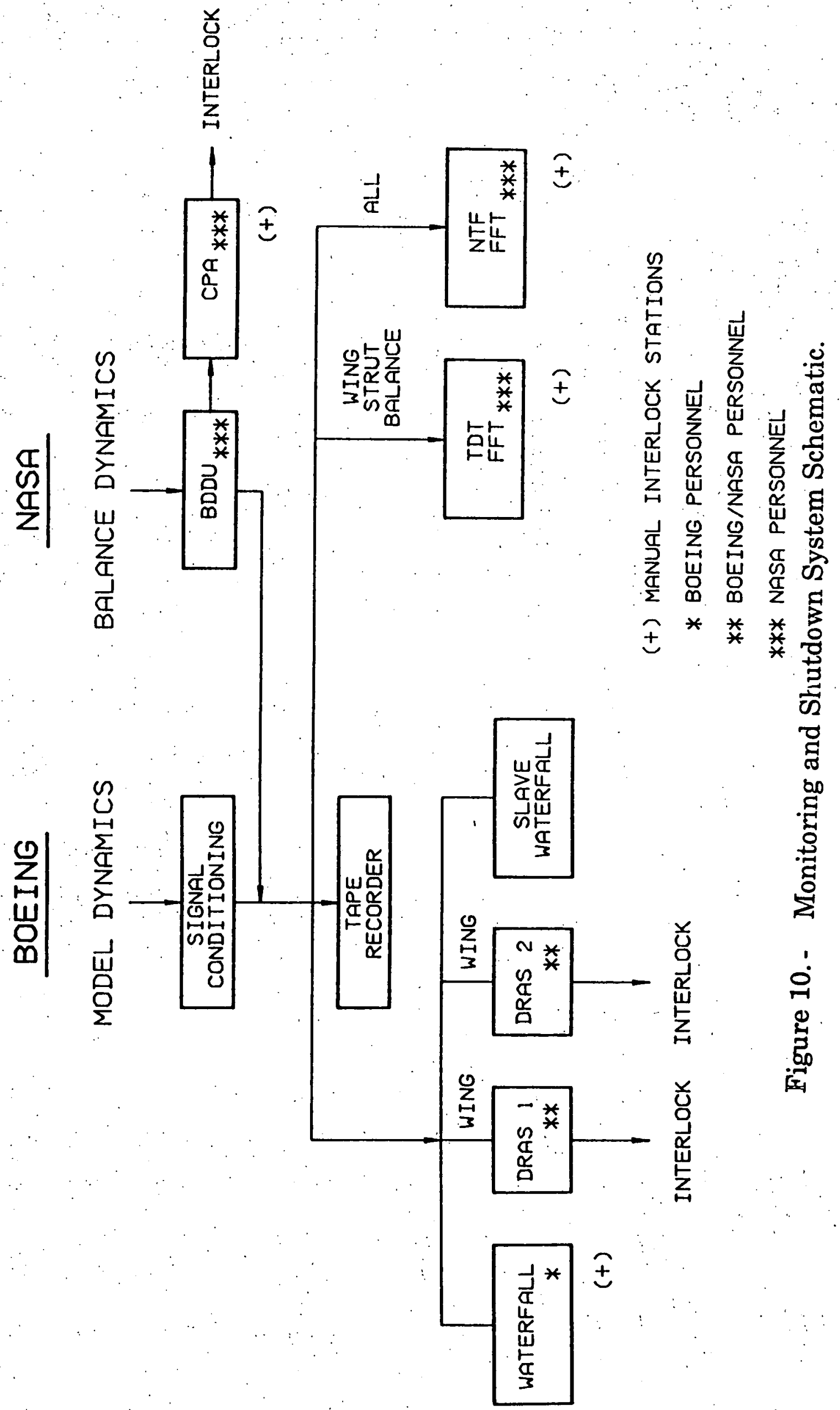




\section{TEST SECTION}

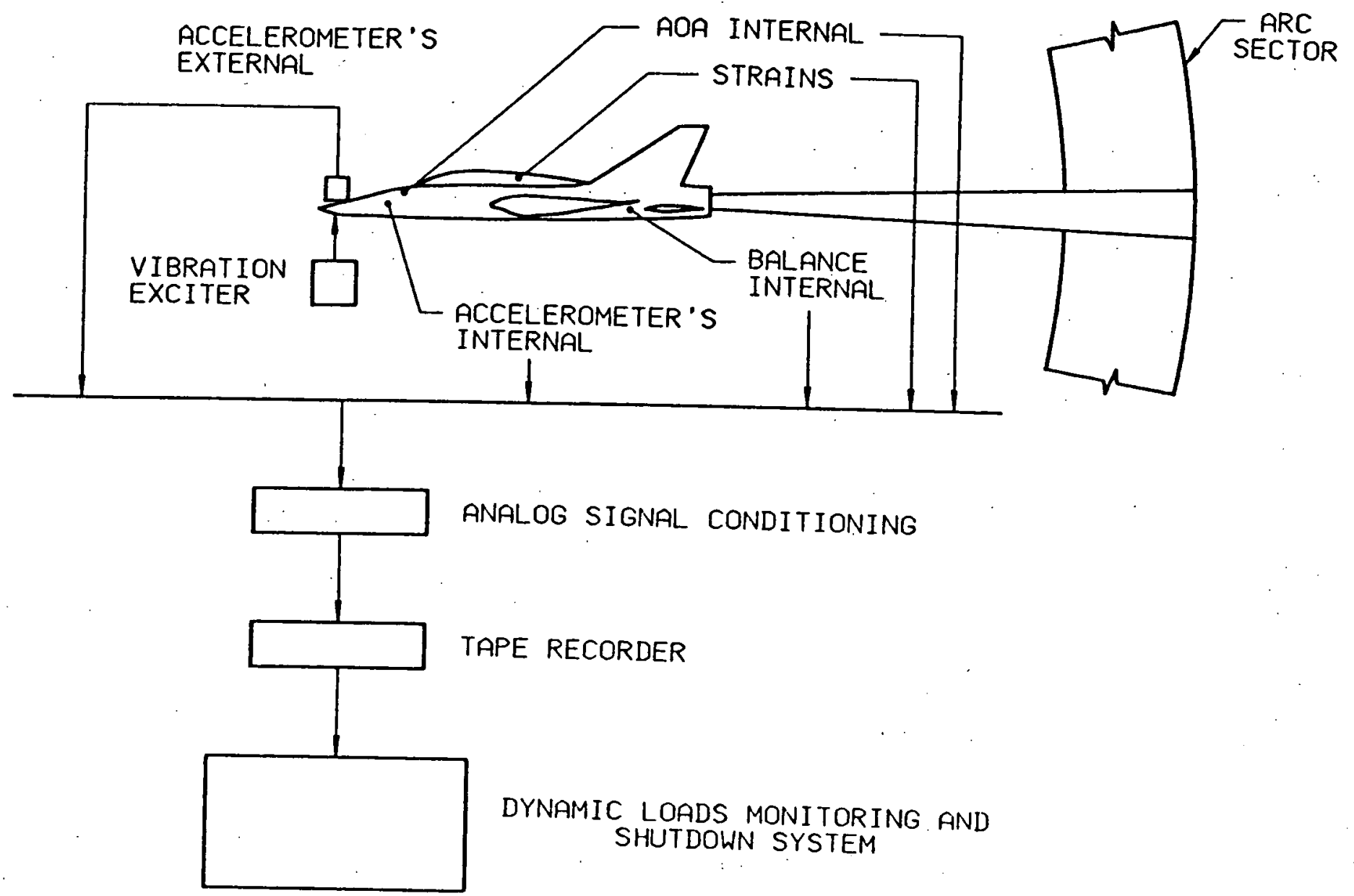

CONTROL ROOM

Figure 11. - Setup for Dynamic Loads Monitoring and Shutdown Systems Checkout. 


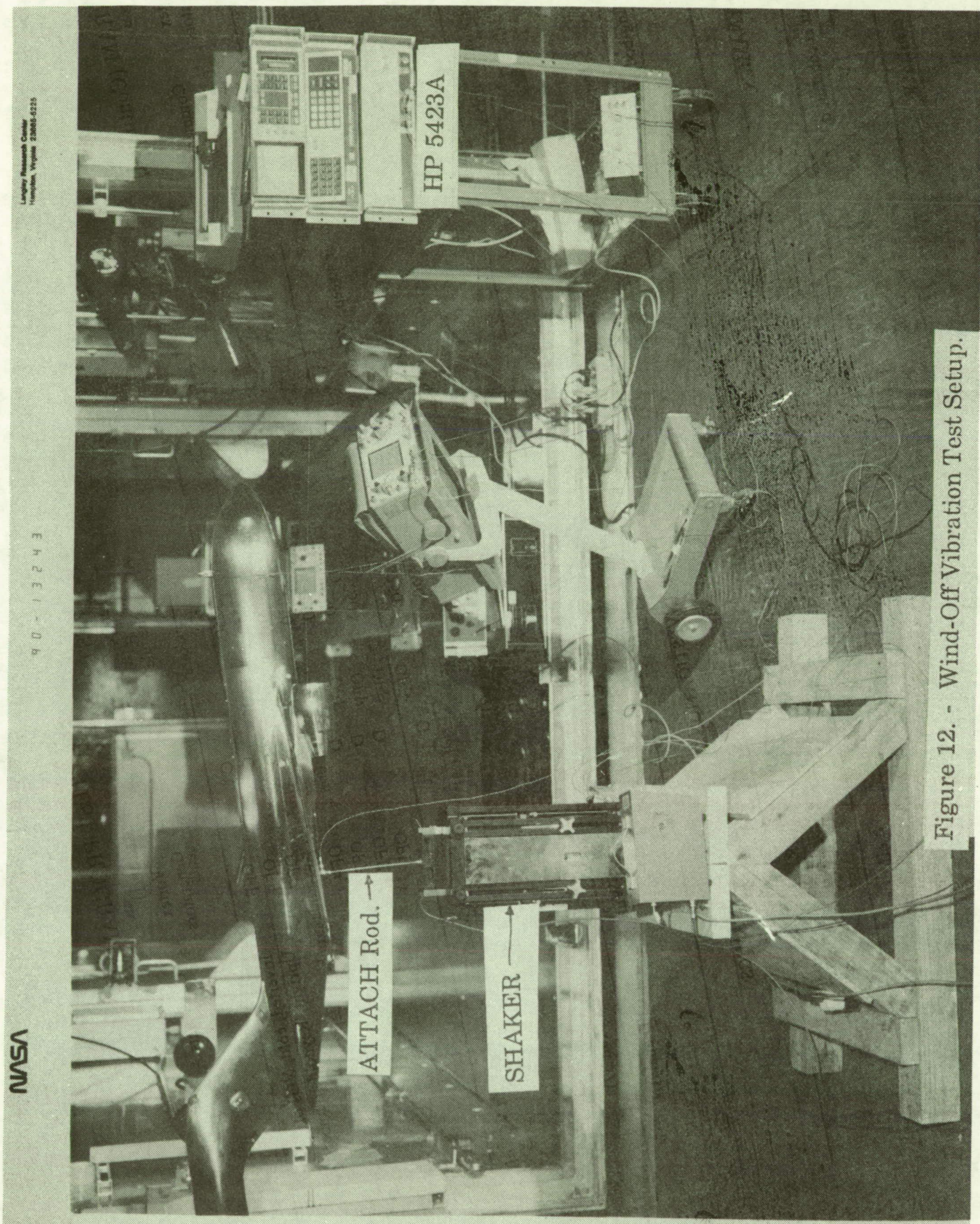




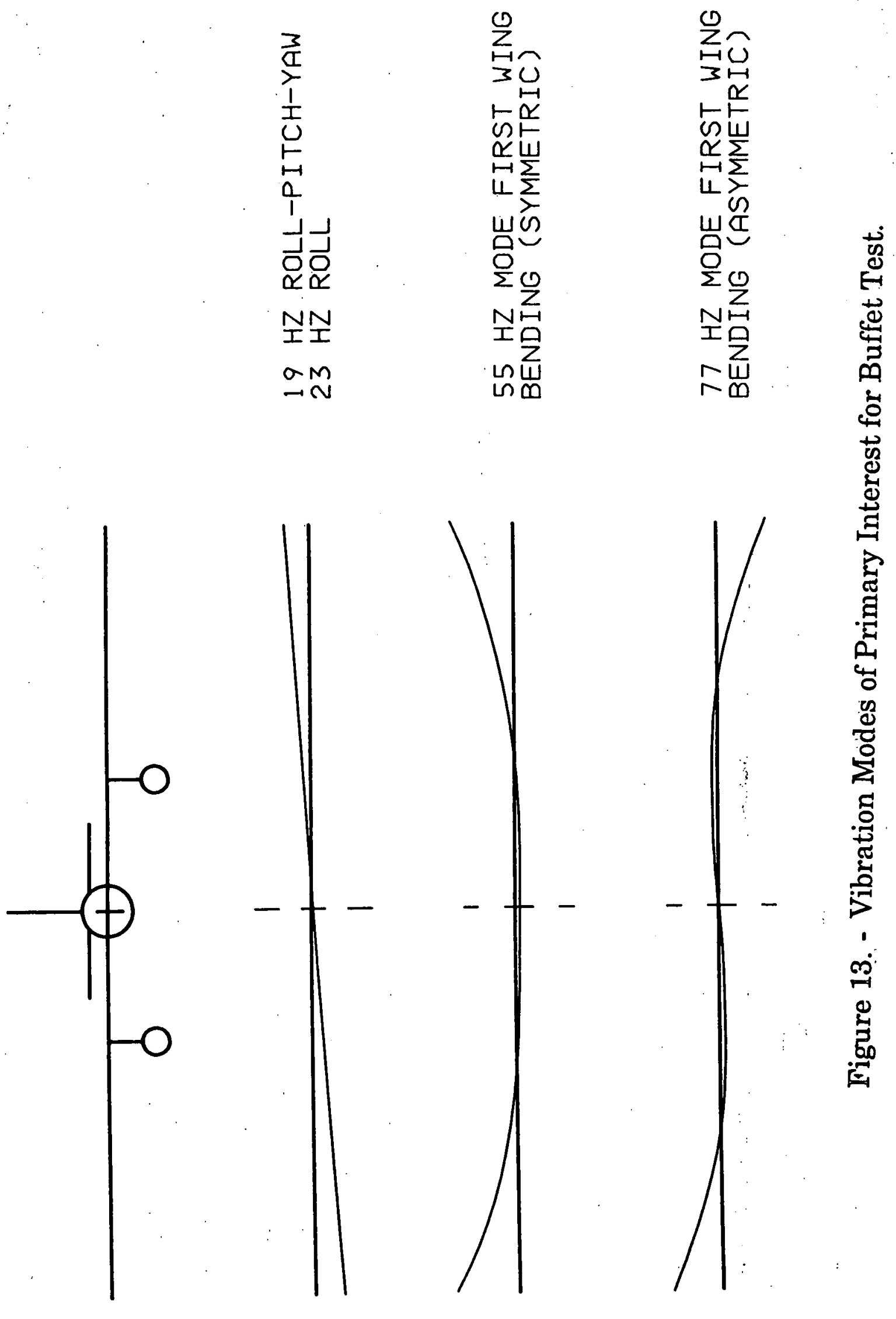




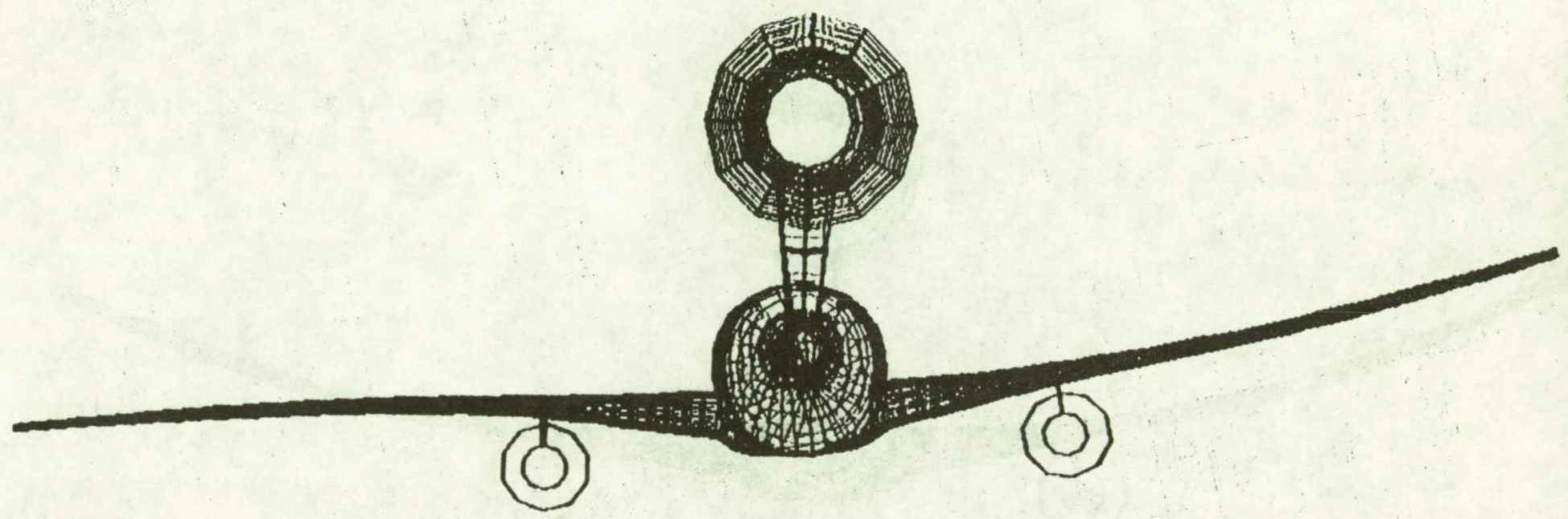

Figure 14. - Finite Element Model of Pure Roll Mode. (20 HZ) 


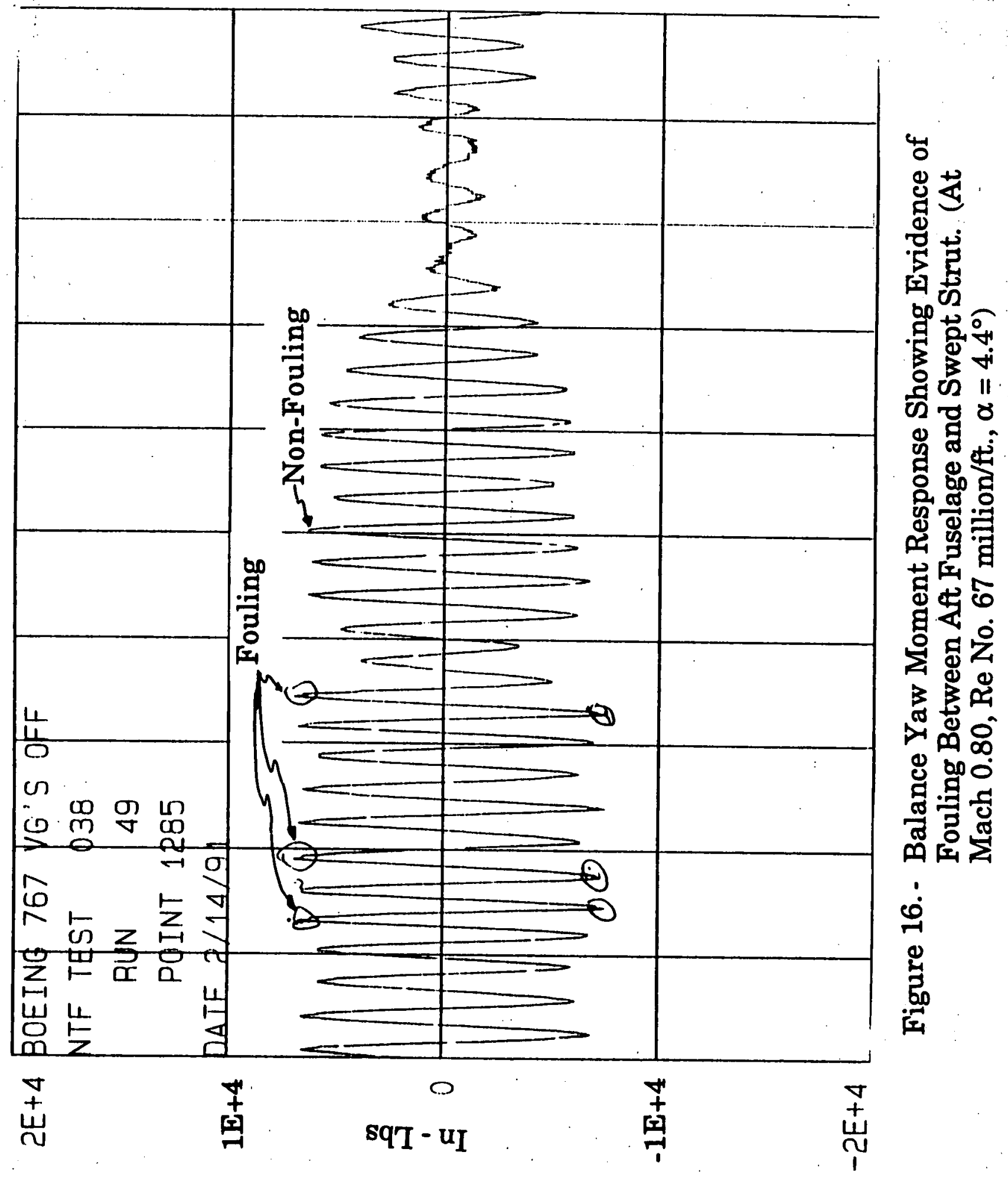


767 Test 101B Balance -Shaking Model 10 Jan 1991

Plot of Yaw \& Side Balance Channels

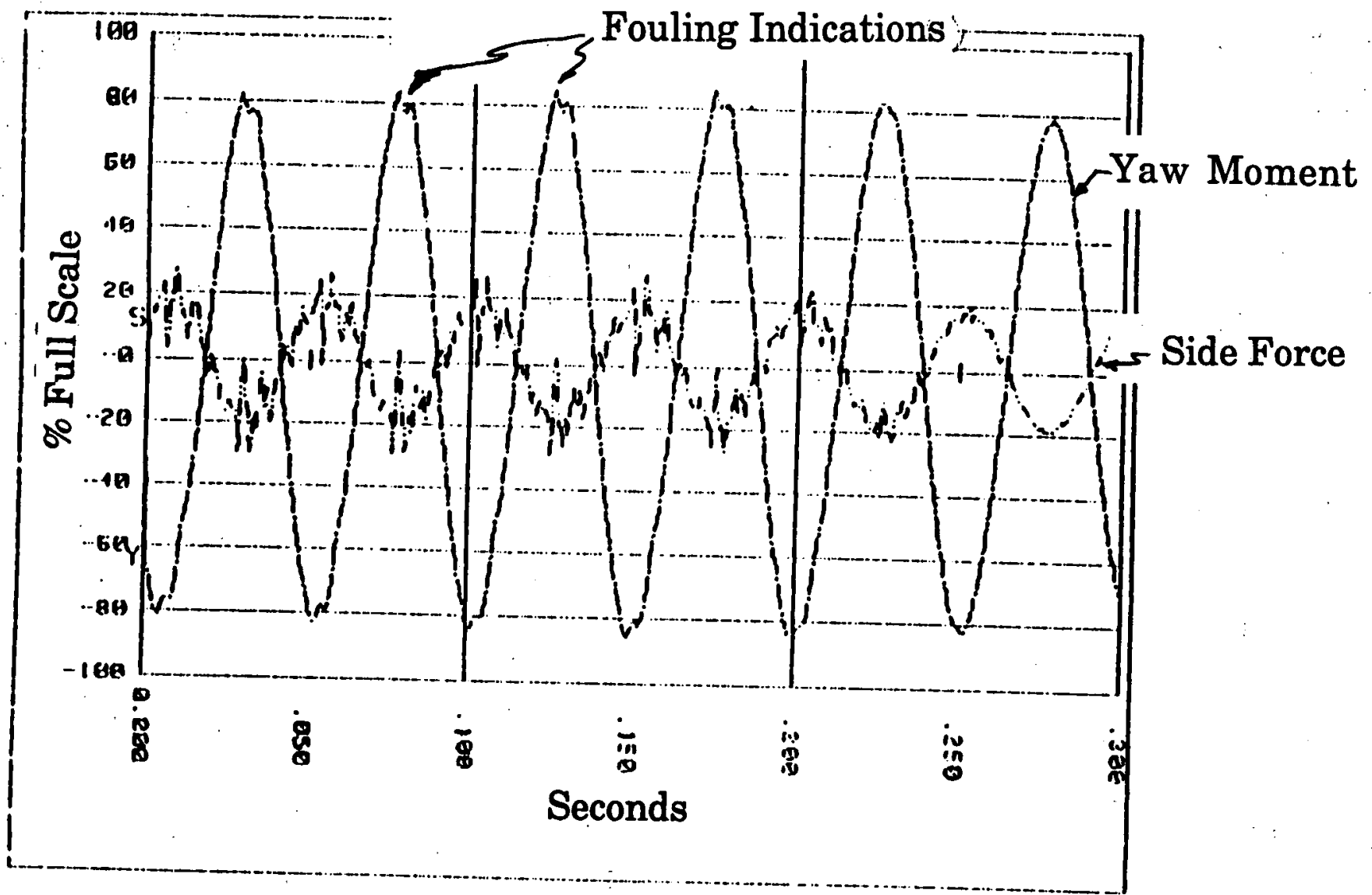

Figure 17.- Balance Yaw Moment and Side Force Response from Forced Vibration Tests Mlustrating Fouling Between Aft Fuselage and Swept Strut. 


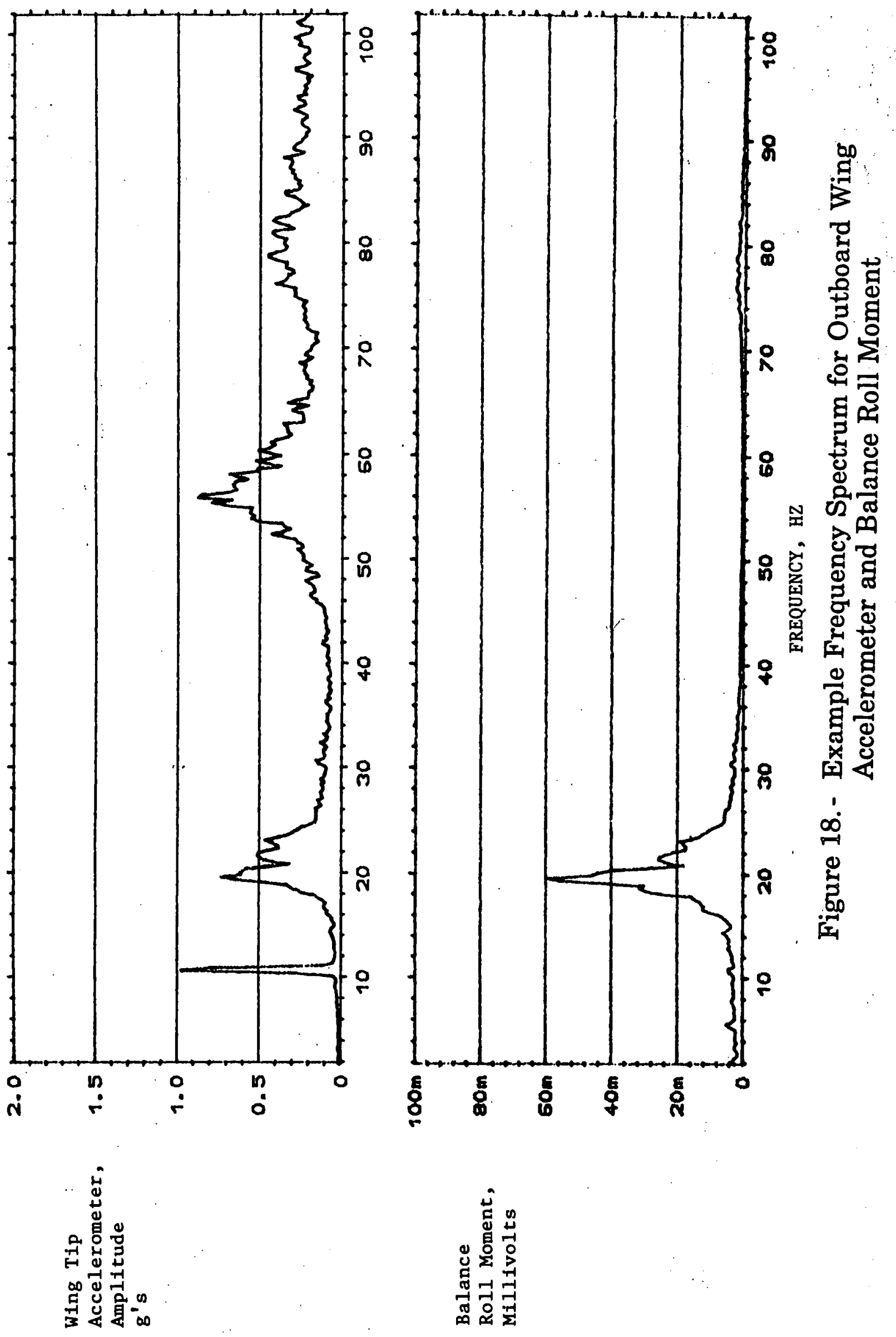




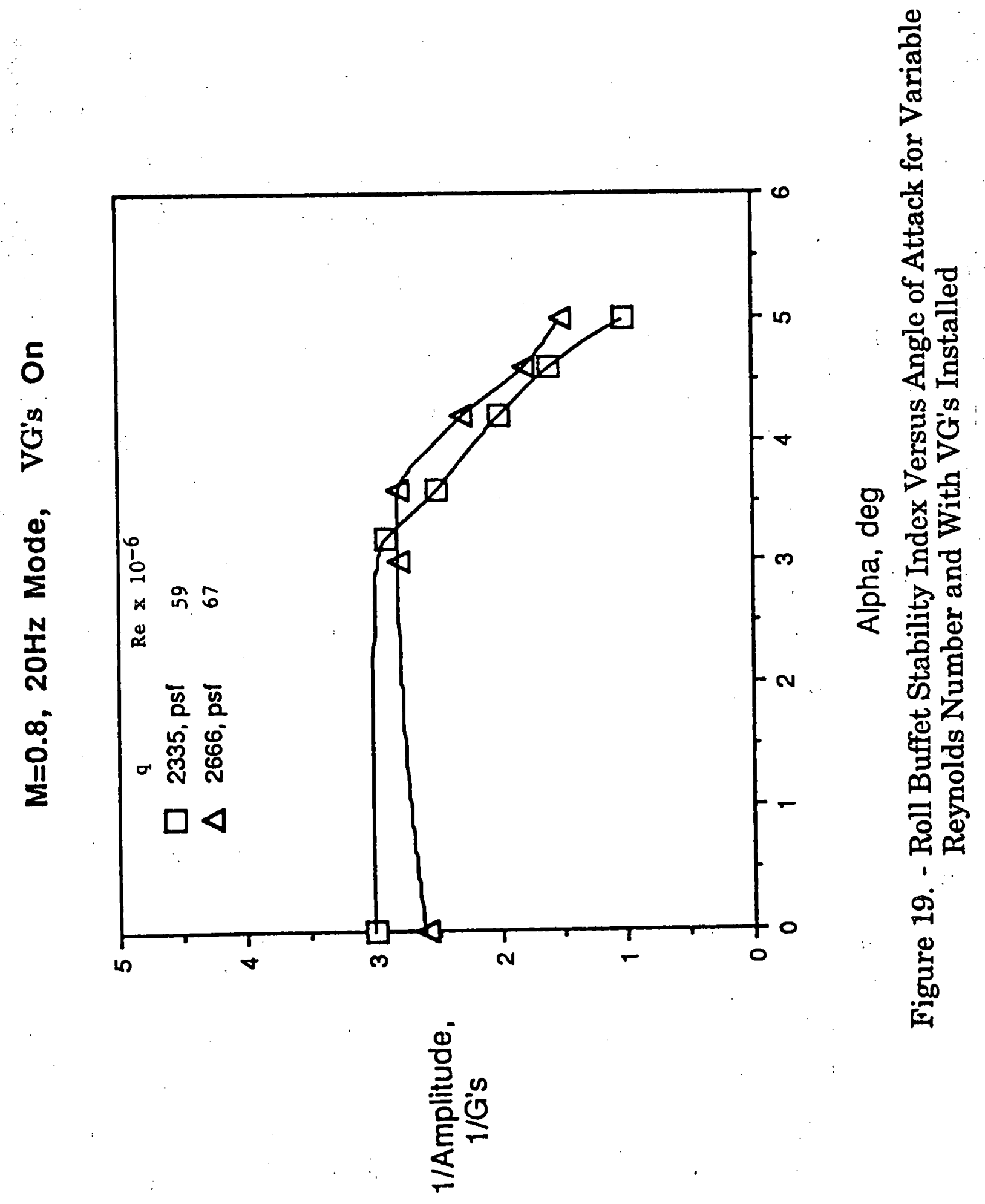




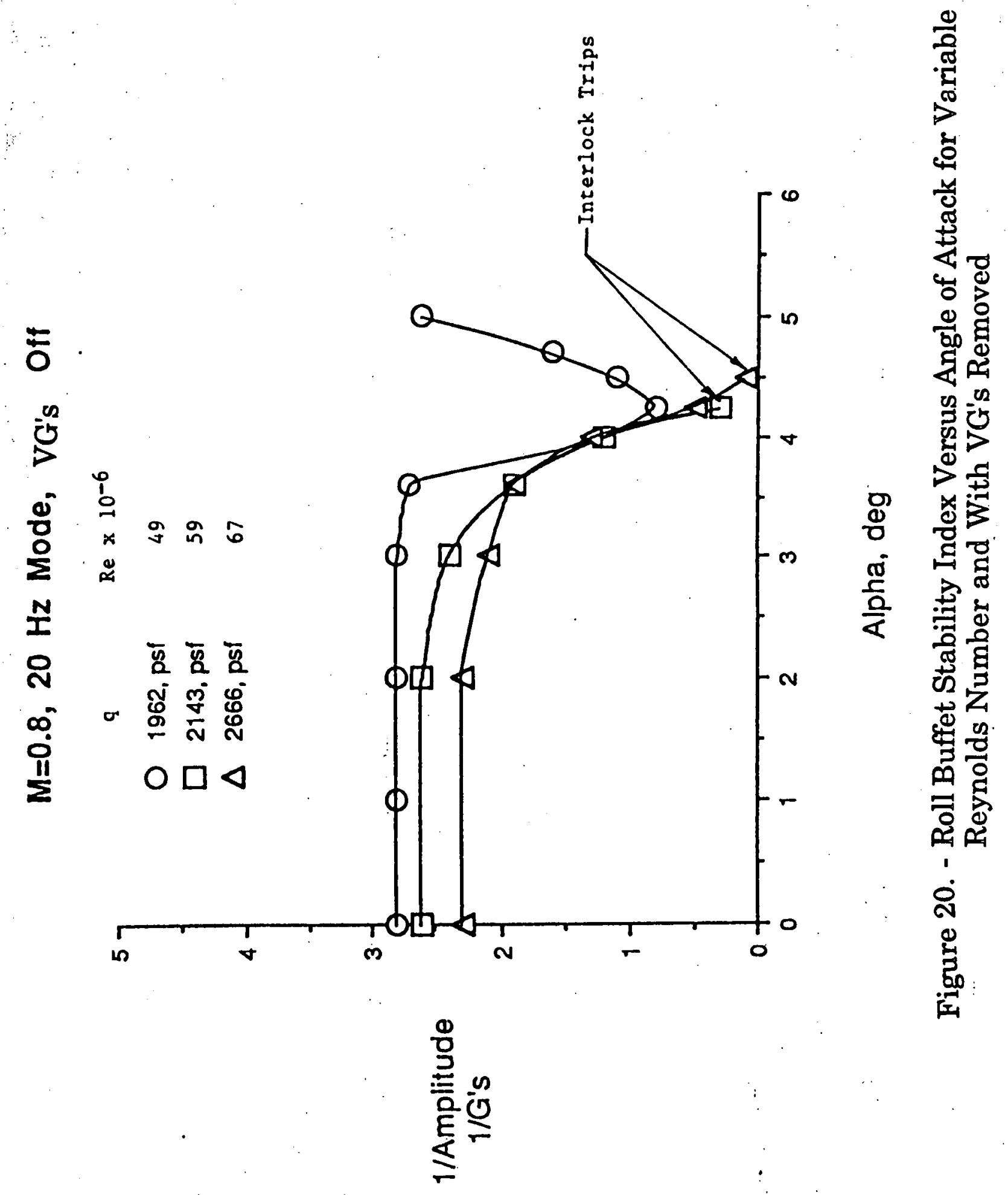




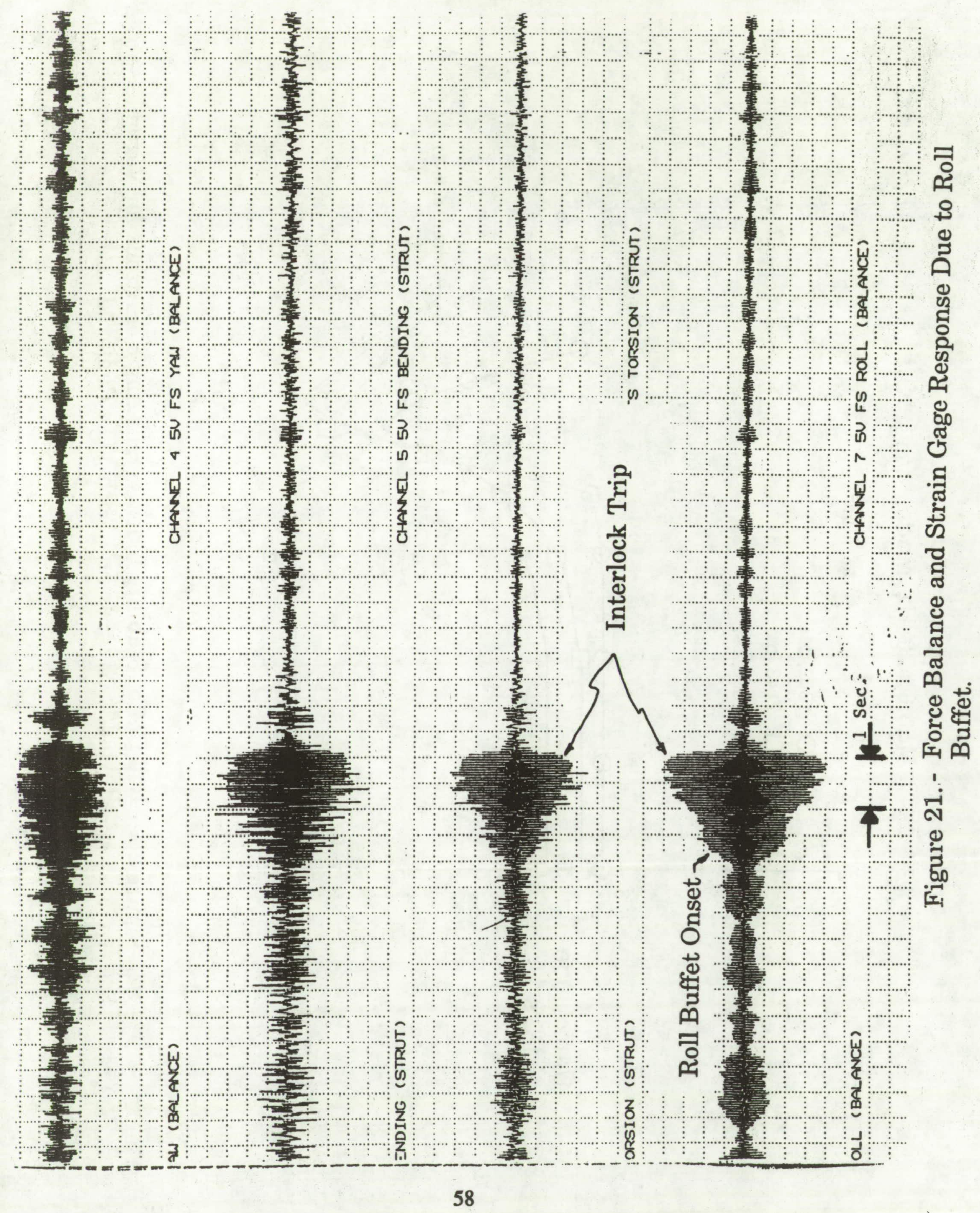




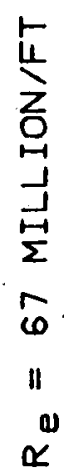
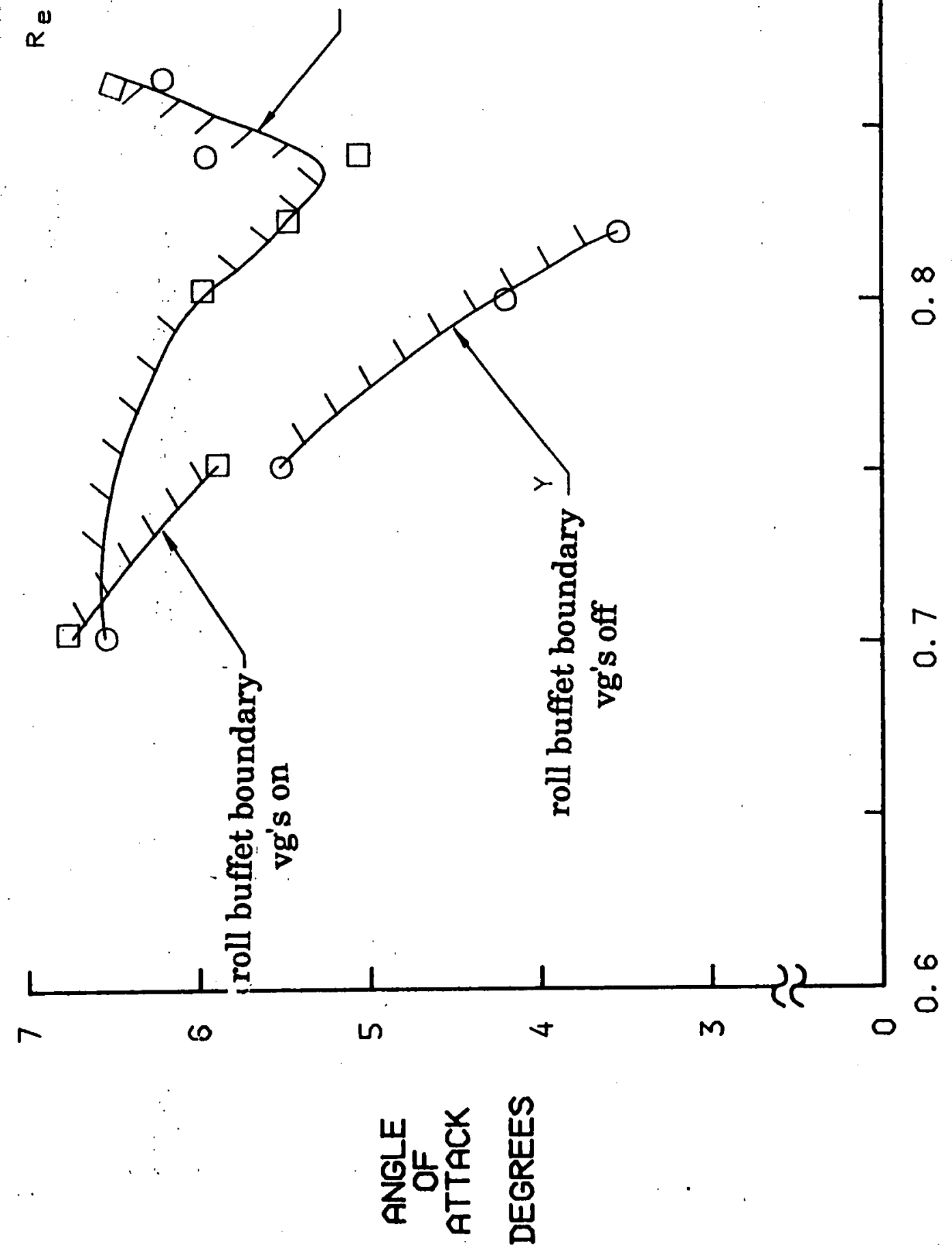

岌 z

@

○ $\square$

능

包

정

نُ

I

ลั

晃 


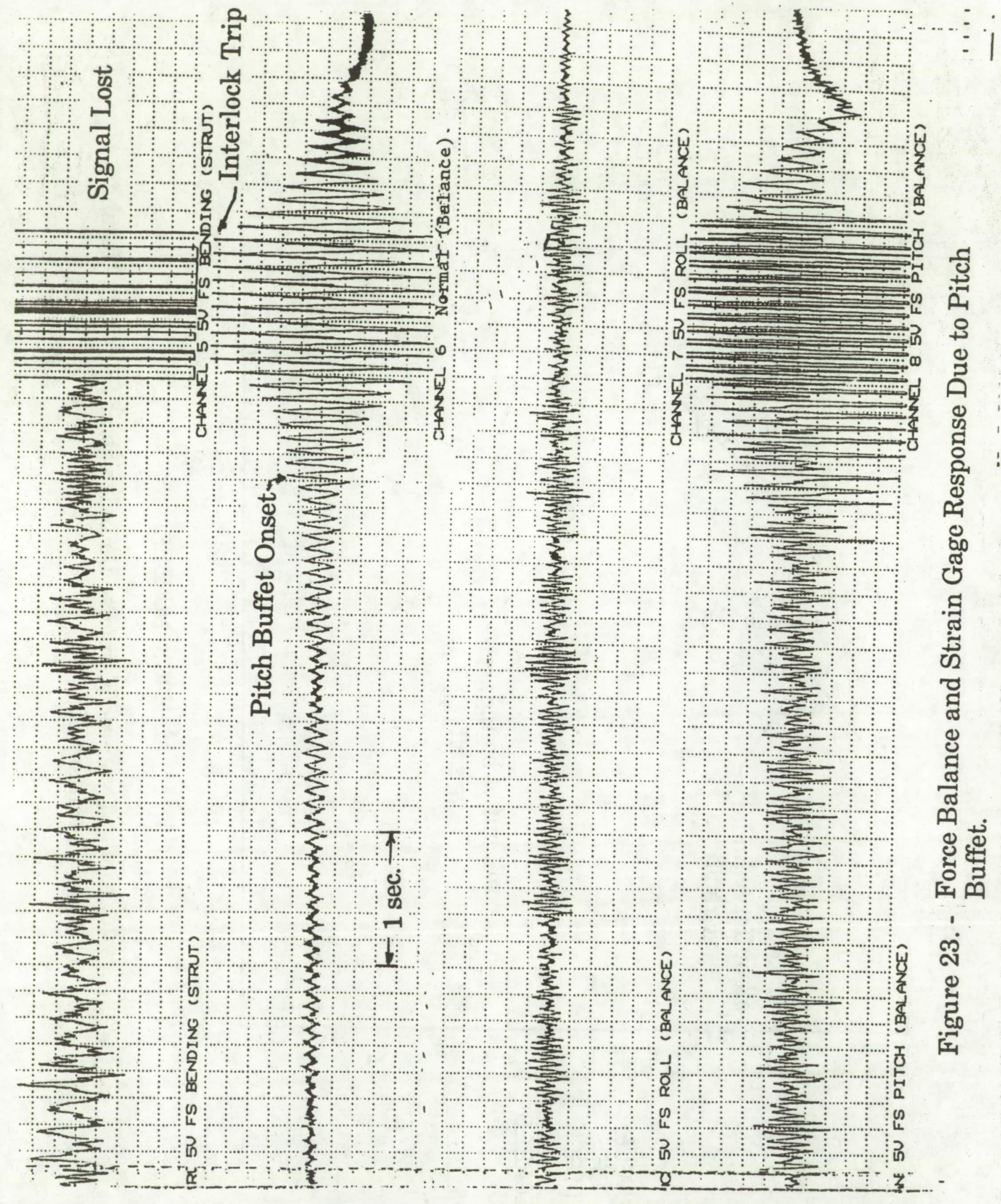


Public reporting burden for this collection of information is est mated to average i hour per response. including the time for reviewing instructions, searching existing. data sources, gathering and maintaining the data needed, and completing and reviewing the collection of information. Send comments regarding this burden estimate or any other aspect of this collection of information. Davis Highway, Suite 1204. Arlington, VA 22202-4302, and to the Office of Management and Budget, Paperwork Reduction Project (0704-0 188), Washington, OC 20503.

\begin{tabular}{|l|l|l} 
1. AGENCY USE ONLY (Leave blank) & $\begin{array}{l}\text { 2. REPORT DATE } \\
\text { July 1992 }\end{array}$ & $\begin{array}{l}\text { 3. REPORT TYPE AND DATES COVERED } \\
\text { Contractor Report }\end{array}$
\end{tabular}

4. TITLE AND SUBTITLE

Buffet Test in the National Transonic Facility

\section{FUNDING NUMBERS}

NCC $1-141$

$505-59-85-01$

6. AUTHOR(S)

Clarence P. Young, Jr., Dennis W. Hergert,

Thomas $W$. Butler, and Fred M. Herring

7. PERFORMING ORGANIZATION NAME(S) AND ADDRESS(ES)

North Carolina State University

Rale1gh, North Carolina 27695-7910 and

The Boeing Company

Seattle, Washington 98124

9: SPONSORING / MONITORING AGENCY NAME(S) AND ADDRESS(ES)

National' Aeronautics and Space Administration

Langley Research Center

Hampton, VA 23665-5225

8. PERFORMING ORGANIZATION REPORT NUMBER

10. SPONSORING / MONITORING AGENCY REPORT NUMBER

NASA CR-189595

\section{SUPPLEMENTARY NOTES}

Langley Technical Monitor: Blair B. Gloss

Final Report

12a. OISTRIBUTION/AVAILABILITY STATEMENT

12b. DISTRIBUTION CODE

Unclassified-Unlimited

Subject Category 09

\section{ABSTRACT (Maximum 200 words)}

A buffet test of a commercial transport model was accomplished in the National Transonic Facility at the NASA Langley Research Center. This aeroelastic test was unprecedented for this wind tunnel and posed a high risk to the facility. This paper presents the test results from a structural dynamics and aeroelastic response point of view and describes the activities required for the safety analysis and risk assessment. The test was conducted in the same manner as a flutter test and employed on-board dynamic instrumentation, real time dynamic data monitoring, automatic, and manual tunnel interlock systems for protecting the model. The procedures and test techniques employed for this test are expected to serve as the basis for future aeroelastic testing in the National Transonic Facility. This test program was a cooperative effort between the Boeing Commercial Airplane Company and the NASA Langley Research Center.

\section{SUBJECT TERMS}

Buffet Testing; Test Techniques; Real Time Dynamic Data Monitoring; Cryogenic Wind Tunnel Testing

17. SECURITY CLASSIFICATION OF REPORT

Unclassified

NSN 7540-01-280-5500 OF THIS PAGE

Unclassified
19. SECURITY CLASSIFICATION OF ABSTRACT

Unclassified
15. NUMBER OF PAGES 66

16. PRICE CODE A04

20. LIMITATION OF ABSTRACT Standard Form 298 (Rev. 2-89) Prescribed by ANSI Sid. 239-18 298-102 